\title{
A new window of exploration in the mass spectrum: strong lensing by galaxy groups in the SL2S $\star, \star \star$
}

\author{
M. Limousin ${ }^{1,2,3}$, R. Cabanac ${ }^{1}$, R. Gavazzi ${ }^{4,5}$, J.-P. Kneib ${ }^{2}$, V. Motta ${ }^{6}$, J. Richard ${ }^{7,8}$, K. Thanjavur ${ }^{9}$, G. Foex ${ }^{10}$, \\ R. Pello ${ }^{10}$, D. Crampton ${ }^{11}$, C. Faure ${ }^{12}$, B. Fort ${ }^{4,5}$, E. Jullo ${ }^{2}$, P. Marshall ${ }^{13}$, Y. Mellier ${ }^{4,5}$, A. More ${ }^{2}$, G. Soucail ${ }^{10}$, \\ S. Suyu ${ }^{14}$, M. Swinbank ${ }^{15}$, J.-F. Sygnet ${ }^{4,5}$, H. Tu ${ }^{16,4,5}$, D. Valls-Gabaud ${ }^{17}$, T. Verdugo ${ }^{6}$, and J. Willis ${ }^{9}$ \\ (Affiliations can be found after the references)
}

Received 4 December 2008 / Accepted 25 May 2009

\begin{abstract}
The existence of strong lensing systems with Einstein radii covering the full mass spectrum, from $\sim 1-2^{\prime \prime}$ (produced by galaxy scale dark matter haloes) to $>10^{\prime \prime}$ (produced by galaxy cluster scale haloes) have long been predicted. Many lenses with Einstein radii around 1-2" and above $10^{\prime \prime}$ have been reported but very few in between. In this article, we present a sample of 13 strong lensing systems with Einstein radii in the range $3^{\prime \prime}-8^{\prime \prime}$ (or image separations in the range $6^{\prime \prime}-16^{\prime \prime}$ ), i.e. systems produced by galaxy group scale dark matter haloes. This group sample spans a redshift range from 0.3 to 0.8 . This opens a new window of exploration in the mass spectrum, around $10^{13}-10^{14} M_{\odot}$, a crucial range for understanding the transition between galaxies and galaxy clusters, and a range that have not been extensively probed with lensing techniques. These systems constitute a subsample of the Strong Lensing Legacy Survey (SL2S), which aims to discover strong lensing systems in the Canada France Hawaii Telescope Legacy Survey (CFHTLS). The sample is based on a search over 100 square degrees, implying a number density of $\sim 0.13$ groups per square degree. Our analysis is based on multi-colour CFHTLS images complemented with Hubble Space Telescope imaging and ground based spectroscopy. Large scale properties are derived from both the light distribution of elliptical galaxies group members and weak lensing of the faint background galaxy population. On small scales, the strong lensing analysis yields Einstein radii between $2.5^{\prime \prime}$ and $8^{\prime \prime}$. On larger scales, strong lens centres coincide with peaks of light distribution, suggesting that light traces mass. Most of the luminosity maps have complicated shapes, implying that these intermediate mass structures may be dynamically young. A weak lensing signal is detected for 6 groups and upper limits are provided for 6 others. Fitting the reduced shear with a Singular Isothermal Sphere, we find $\sigma_{\mathrm{SIS}} \sim 500 \mathrm{~km} \mathrm{~s}^{-1}$ with large error bars and an upper limit of $\sim 900 \mathrm{~km} \mathrm{~s}^{-1}$ for the whole sample (except for the highest redshift structure whose velocity dispersion is consistent with that of a galaxy cluster). The mass-to-light ratio for the sample is found to be $M / L_{i} \sim 250$ (solar units, corrected for evolution), with an upper limit of 500. This compares with mass-to-light ratios of small groups (with $\sigma_{\mathrm{SIS}} \sim 300 \mathrm{~km} \mathrm{~s}^{-1}$ ) and galaxy clusters (with $\sigma_{\mathrm{SIS}}>1000 \mathrm{~km} \mathrm{~s}^{-1}$ ), thus bridging the gap between these mass scales. The group sample released in this paper will be complemented with other observations, providing a unique sample to study this important intermediate mass range in further detail.
\end{abstract}

Key words. gravitational lensing: cosmology: large-scale structure of Universe

\section{Introduction}

\subsection{Galaxy groups and cosmology}

Galaxy groups are believed to play a key role in the formation and evolution of structures in the Universe. They contain the majority of the galaxies in the Universe, and within a hierarchical framework, they span the regime between individual galaxies and massive galaxy clusters, making them cosmologically significant. They are also more varied in their properties than

* Appendix A is only available in electronic form via http://www . aanda.org

$\star \star$ Based on observations obtained with MegaPrime/MegaCam, a joint project of CFHT and CEA/DAPNIA, at the Canada-France-Hawaii Telescope (CFHT) which is operated by the National Research Council (NRC) of Canada, the Institut National des Sciences de l'Univers of the Centre National de la Recherche Scientifique (CNRS) of France, and the University of Hawaii. This work is based in part on data products produced at TERAPIX and the Canadian Astronomy Data Centre as part of the Canada-France-Hawaii Telescope Legacy Survey, a collaborative project of NRC and CNRS. Based on observations made with the NASA/ESA Hubble Space Telescope, obtained at the Space Telescope Science Institute, which is operated by the Association of Universities for Research in Astronomy, Inc., under NASA contract NAS 5-26555. These observations are associated with programs 10876 and 11289. galaxy clusters, as demonstrated by comparisons between various scaling relations in galaxy groups to those in galaxy clusters. This indicates that galaxy groups are probably not a homogeneous class of objects, e.g. they cannot be considered simple scaled-down versions of galaxy clusters. Detailed studies of this intermediate regime of the mass spectrum $\left(\sim 10^{13}-10^{14} M_{\odot}\right)$ are needed to understand better the physical processes behind formation of galaxy groups and how galaxy groups participate to structure formation and evolution.

Properties of low and intermediate redshift galaxy groups $(z<0.5)$ have been studied using X-ray and optical tracers (e.g. Helsdon \& Ponman 2000, 2003; Osmond \& Ponman 2004; Willis et al. 2005; Finoguenov et al. 2007; Rasmussen \& Ponman 2007; Mamon 2007; Faltenbacher \& Mathews 2007; Gastaldello et al. 2007; Yang et al. 2008; van den Bosch et al. 2008; Sun et al. 2009, and references therein). Galaxy groups have also been studied numerically (e.g. D’Onghia et al. 2005; Sommer-Larsen 2006, and references therein).

\subsection{Strong lens statistics}

Figure 1 shows the predicted contributions of different types of haloes in the image separation distributions (from Oguri 2006, Fig. 9) extracted from N-body simulations, where $\theta$ corresponds 


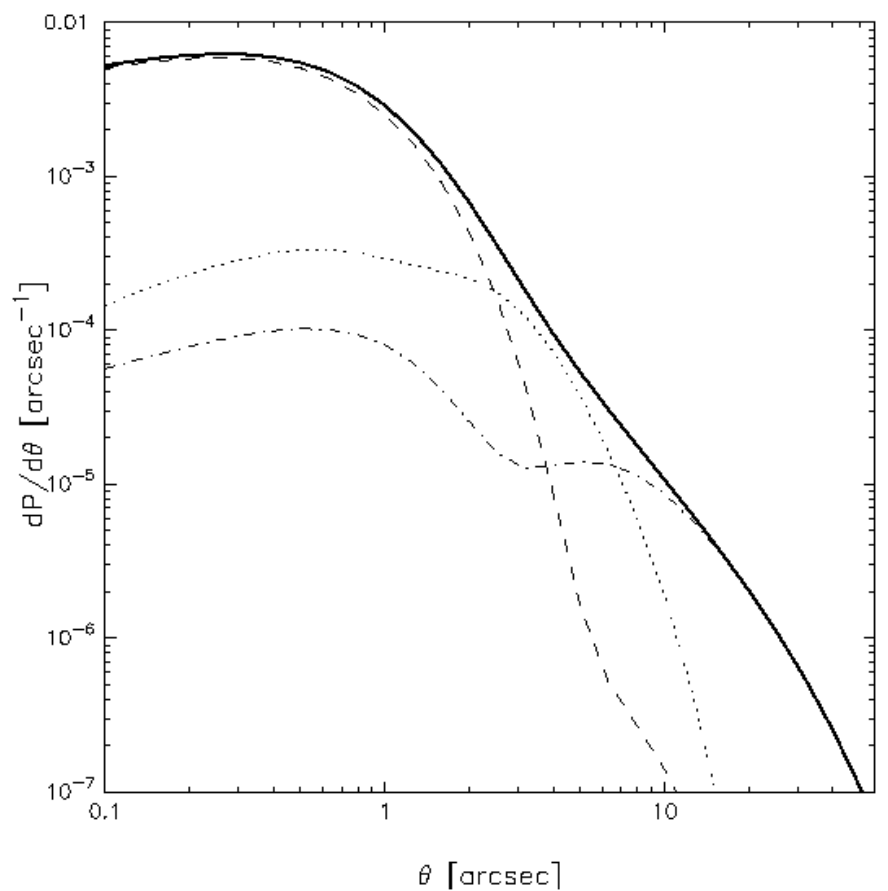

Fig. 1. The contributions of different types of haloes on the image separation distribution, from Oguri (2006): galaxy (dashed); groups (dot) and clusters (dot-dashed). The sum of distributions of three types is shown by thick solid line.

to about twice the Einstein radius of the lens ${ }^{1}$. This plot shows that there are large overlaps among the halo types, but also that some scales are dominated by different regimes: on small scales, the distribution is dominated by galaxy-scale dark-matter haloes, with $R_{\mathrm{E}}<2^{\prime \prime}$, and on larger scales, by cluster-scale dark-matter haloes, with $R_{\mathrm{E}}>10^{\prime \prime}$. In between, the distribution is dominated by haloes generating strong lensing deflection of radii between $\sim 3^{\prime \prime}$ and $\sim 8^{\prime \prime}$, whose mass is in the range $10^{13}-10^{14} M_{\odot}$, i.e. by group-scale dark-matter haloes.

Hence according to simulations, strong lenses from intermediate mass systems should exist in a $\Lambda$ CDM Universe. Until now, only a few cases have been detected observationally in the SDSS, which we discuss in Sect. 7.6.

Strong lensing deflector: In this paper, we refer to a "deflector" as the foreground mass distribution giving rise to multiple images, and we focus on its central part. Specifically, a strong lensing deflector refers to the area of the foreground mass distribution enclosed by the multiple images. This region can be populated by more than a single galaxy on scales smaller than $R_{\mathrm{E}}$, as it is the case for some of the galaxy groups reported in this work. In order to avoid confusion, we use the term deflector instead of lensing galaxy. This is particularly important for group scale deflectors since their mass may no longer be associated to a single galaxy. This definition is well adapted to most of the groups studied in this paper, where the multiple images identification is clear and therefore the region enclosed by these images is well defined. However, this is not the case for three of the groups presented in this paper.

\footnotetext{
${ }^{1}$ In this paper, we will use the Einstein radius $R_{\mathrm{E}}$ to characterise the lenses.
}

\subsection{Strong lensing in and by a galaxy group}

We shall emphasise the distinction between strong lensing in a galaxy group and by a galaxy group. Strong lensing in a galaxy group corresponds to the observation of a strong lensing feature, with a typical Einstein radius of $\sim 2{ }^{\prime \prime}$, generated by a galaxy group member. Since the high-density environment is likely to enhance the strong galaxy-galaxy lensing cross section (see Kovner 1987; Möller et al. 2002; Fassnacht et al. 2006; Oguri et al. 2005; King 2007; Newton et al. 2008, but see Faure et al. 2008, in the CosMos field), many strong lensing systems in groups (or clusters) have been reported (e.g. Kundic et al. 1997; Fassnacht \& Lubin 2002; Morgan et al. 2005; Williams et al. 2006; Momcheva et al. 2006; Auger et al. 2007; Tu et al. 2008; Auger et al. 2008; Grillo et al. 2008; Treu et al. 2009; Inada et al. 2009). If the creation of multiple images is boosted by external shear and convergence from a smooth group component, these strong lensing systems are, to first order, generated by a galaxyscale dark-matter halo populated by a single galaxy (most of the time an elliptical galaxy, but note that efforts are currently underway to find lenses generated by haloes populated by spiral galaxies, see Feron et al. 2008; Trott et al. 2008).

On the other hand, strong lensing by a galaxy group corresponds to the observation of strongly lensed features with a typical Einstein radius between $\sim 3^{\prime \prime}$ and $\sim 8^{\prime \prime}$. This translates into a projected mass enclosed within this radius around $\sim 10^{12.5}$ $10^{13} M_{\odot}$, i.e. characteristic of a group-scale dark-matter halo. Note that the highest limit of $8^{\prime \prime}$ adopted here is somewhat arbitrary and may be considered by others as a characteristic size of a poor cluster.

\subsection{Galaxy groups in the strong lensing legacy survey}

A straightforward method for finding lens systems is to identify them directly within existing multi-band wide-field imaging surveys. For this purpose, we have started to explore systematically the Canada France Hawaii Telescope Legacy Survey (CFHTLS), using a dedicated automatic search procedure optimised for the detection of arcs. This subsample of the CFHTLS forms the Strong Lensing Legacy Survey (SL2S, Cabanac et al. 2007). This sample is not biased towards high masses by any prior X-ray selection and because of the wide area covered, cosmic variance is neglected and the resulting sample of strong lenses is assumed to be representative of what can be found in the Universe. However, selection biases inherent to our detection technique do exist and will be addressed in a forthcoming publication using numerical simulations. The SL2S sample probes a wide range of masses and a wide range of redshifts for the lenses. Thanks to unsurpassed combined depth, area and image quality of the CFHTLS, we have uncovered a new population of lenses, with Einstein radii between $\sim 3^{\prime \prime}$ and $\sim 8^{\prime \prime}$, i.e. generated by galaxy-group scale-dark matter haloes. This new population effectively bridges the gap between single galaxies and massive clusters, opening a new window of exploration in the mass spectrum. The purpose of this paper is to present the first representative sample of such lenses. Note that this sample is not statistically complete since the survey is not complete yet and that we do not understand properly our selection function.

This paper is organised as follows: data used in this work are presented in Sect. 2. Sections 3-5 present the methodology of the systematic analysis we apply on each group. Results are presented in Sect. 6 and discussed in Sect. 7. In the Appendix, each group is described individually, with some images. All our results are scaled to a flat, $\Lambda$ CDM cosmology 
with $\Omega_{\mathrm{M}}=0.3, \Omega_{\Lambda}=0.7$ and a Hubble constant $\mathrm{H}_{0}=$ $70 \mathrm{~km} \mathrm{~s}^{-1} \mathrm{Mpc}^{-1}$. All images are aligned with WCS coordinates, i.e. north is up, east is left. Magnitudes are given in the AB system.

\section{Data}

In this section, we present the various data sets used in this work. Multicolour imaging from the CFHTLS constitutes the basis of our analysis and is complemented with ongoing HST imaging and ongoing ground-based spectroscopy.

\subsection{The Canada-France-Hawaii telescope legacy survey}

\subsubsection{Description}

The CFTHLS is a major photometric survey of more than 450 nights over 5 years (started on June 1st, 2003, ending 2008) using the wide field imager MEGAPRIME which covers $\sim 1$ square degree on the sky, with a pixel size of $0.186^{\prime \prime}$. The project is comprehensively described in http://www. cfht.hawaii. edu/Science/CFHLS/ and links therein. The CFHTLS has two components aimed at extragalactic studies: a very Deep component made of 4 pencil-beam fields of $1 \mathrm{deg}^{2}$ and a Wide component made of 3 mosaics covering $170 \mathrm{deg}^{2}$ in total, both in 5 broadband filters. The data are pre-reduced at CFHT with the Elixir pipeline ${ }^{2}$ which removes the instrumental artifacts in individual exposures. The CFHTLS images are then evaluated, astrometrically calibrated, photometrically inter-calibrated, resampled and stacked by the Terapix group at the Institut d'Astrophysique de Paris (IAP) and finally archived at the Canadian Astronomy Data Centre (CADC). Terapix also provides weightmap images, quality assessments meta-data for each stack as well as mask files that mask straylight, saturated stars and defects on each image.

\subsubsection{Terapix release T0004}

The SL2S group sample presented here is based on the T0004 release (July 2007), corresponding to data obtained between the Spring 2003 and the spring 2007. A detailed description of this release is given at the Terapix web site ${ }^{3}$ and CADC site ${ }^{4}$. The T0004 release includes $120 \mathrm{deg}^{2}$ of stacked fields in the Wide survey observed in broadband $g^{\prime}, r^{\prime}$ and $i^{\prime}$ filters, and a stack of the 35 Wide and 4 Deep fields in the 5 bands, for a total area of $124 \mathrm{deg}^{2}$, or ca. $110 \mathrm{deg}^{2}$ of unmasked area, i.e. area not contaminated by instrumental artefacts from bright stars (internal reflections, bleeding). Table 1 summarises the main characteristics of the T0004 release. The 4 Deep fields are much deeper, with a seeing ranging from $0.9^{\prime \prime}$ in $u^{*}$ to $0.7^{\prime \prime}$ in $z^{\prime}$. Because of the observing strategy, the Wide survey image quality is prone to large variations from $1^{\prime \prime}$ to $0.6^{\prime \prime}$ seeing in all bands. Nevertheless, the Wide survey is more suited to our strong lensing selection processes, because of its wide angular coverage.

\subsection{Space based imaging}

The strong lensing features detected from ground-based images have been observed with the Hubble Space Telescope (HST)

\footnotetext{
${ }^{2}$ http://www.cfht.hawaii.edu/Instruments/Elixir/

${ }^{3}$ http://terapix.iap.fr

${ }^{4}$ http://www2 . cadc-ccda.hia-iha.nrc-cnrc.gc.ca/cfht/ T0004.html
}

Table 1. CFHTLS: Terapix T0004 release (July 2007).

\begin{tabular}{lccccc}
\hline \hline Deep fields & \multicolumn{5}{c}{ AB Magnitudes Limits } \\
& $u^{*}$ & $g^{\prime}$ & $r^{\prime}$ & $i^{\prime}$ & $z^{\prime}$ \\
D1-25 & 26.7 & 27.5 & 27.5 & 27.3 & 26.0 \\
D2-25 & 25.6 & 27.3 & 27.3 & 27.0 & 25.6 \\
D3-25 & 26.6 & 27.5 & 27.4 & 27.2 & 26.1 \\
D4-25 & 26.9 & 27.5 & 27.5 & 27.1 & 26.0 \\
D1-25 seeing & 0.93 & 0.93 & 0.75 & 0.74 & 0.71 \\
D2-25 seeing & 0.83 & 0.83 & 0.77 & 0.71 & 0.72 \\
D3-25 seeing & 0.89 & 0.89 & 0.77 & 0.73 & 0.64 \\
D4-25 seeing & 0.86 & 0.86 & 0.77 & 0.72 & 0.71 \\
Wide fields & AB Average Magnitudes Limits \\
W1 & 25.8 & 26.5 & 26.0 & 25.8 & 25 \\
W2 & 25.8 & 26.5 & 26.0 & 25.8 & 25 \\
W3 & 25.8 & 26.5 & 26.0 & 25.8 & 25 \\
W4 & 25.8 & 26.5 & 26.0 & 25.8 & 25 \\
W1 area (unmasked) & \multicolumn{5}{c}{$44(39) \mathrm{deg}^{2}$} \\
W2 area (unmasked) & \multicolumn{5}{c}{$40(16) \mathrm{deg}^{2}$} \\
W3 area (unmasked) & \multicolumn{5}{c}{$16(13) \mathrm{deg}^{2}$} \\
W4 area (unmasked) & \\
\end{tabular}

For a point source $S / N=5\left(A B\right.$ to Vega $u^{*}-0.35, g^{\prime}+0.09, r^{\prime}-0.17$, $\left.i^{\prime}-0.40, z^{\prime}-0.55\right)$

for 11 of the 13 groups presented in this paper. Observations were done in snapshot mode (C15 and C 16, P.I. Kneib, ID 10876 and 11289). 3 groups have been imaged in three bands with the ACS camera (F814, F606 and F475), and the remaining in the F606 band only with the WFPC2 camera. Space-based imaging generally allows one to better resolve the strong lensing systems we are interested in.

\subsection{Spectroscopy}

Various ongoing spectroscopic campaigns are targeting both the galaxy group members as well as the multiply imaged systems. We use the following facilities:

1. FORS 2 on VLT: resolution of $600 \mathrm{RI}$, exposure $2800 \mathrm{~s}$, with $\Delta \Lambda$ from 5000 to $8000 \AA$;

2. LRIS on Keck: Dichroic 680 and $560 \mathrm{~nm}$, exposure $600 \mathrm{~s}$ and $1200 \mathrm{~s}$ with a slit of $1.5^{\prime \prime}$;

3. Gemini (Program GN-2007A-Q-114): we observed SL2S J14300+5546 on June 2007 using GMOS LS+R400 grating at $7500 \AA$ A during 3750 s.

When available and relevant to this work, we will report some of the results of our spectroscopic campaigns. Details of the observing runs and spectra will be presented in forthcoming publications. For SL2S J14081+5429 and SL2S J22214-0053, we report the spectroscopic redshift measured by the Sloan Digital Sky Survey (SDSS).

\subsection{Photometric redshifts}

Photometric redshifts were estimated for all the groups, based on the magnitudes of the brightest galaxy populating the strong lensing deflector whose coordinates are given in Table 3. Aperture magnitudes were extracted with SEXTRACTOR 2.5 (Bertin \& Arnouts 1996) and photometric redshifts were estimated using the HyperZ software (Bolzonella et al. 2000). For all groups but two (SL2S J14300+5546 and SL2S J14314+5533), 5 photometric bands are available. 
Photometric redshifts, with $3 \sigma$ error bars are given in Table 3 . They can be compared to the spectroscopic measurements available for 9 of the groups. We note that in the case of 4 groups over 9, the spectroscopic redshifts are not consistent with the $3 \sigma$ confidence intervals of the photometric redshifts estimates. However, the difference between each estimate is smaller than 0.03 . This has no influence on the lensing properties of the galaxy groups and therefore does not bias our analysis.

In addition, we have been looking systematically in the NED database at all known sources within $5^{\prime}$ of each strong lensing event to gather any observational data relevant to this study.

\section{Building luminosity maps}

Luminosity maps were created from the $i$-band isophotal magnitudes of all the elliptical galaxies tagged as members of the group. This is the deepest band that best represents the old stellar population of elliptical galaxies. Catalogues of objects positions and magnitudes have been generated using SEXTRACTOR 2.5.

\subsection{Selecting group members}

We build matched $g, r, i$ bands catalogues of all objects with a radius of $5^{\prime}$ of the lens centre. We compute the $r-i$ colour of the brightest galaxy populating the strong lensing deflector. We refer to this quantity in the following as $(r-i)_{0}$. We then consider all galaxies whose $r-i$ colour satisfy:

$(r-i)_{0}-0.15<r-i<(r-i)_{0}+0.15 \& i<\operatorname{mag}_{\lim }(z)$

as possible galaxy group red-sequence members (e.g. Gladders et al. 1998). We miss the faintest group members but the most luminous ellipticals are correctly selected. When spectroscopy of group members is available, we verified that our procedure does select the spectroscopically confirmed group members. A more robust selection will require us to analyse multi-object spectroscopy of the fields.

In order to compare the group luminosity maps across the redshift range, we restrict our group member catalogue to galaxies brighter than a magnitude threshold $\operatorname{mag}_{\text {lim }}(z)$. This magnitude threshold is set by the apparent magnitude of the faintest elliptical member of the highest redshift group (SL2S J22133+0048 at $\left.z_{\text {phot }}=0.83\right)$. That apparent magnitude is converted into a rest-frame absolute luminosity, converted back into a redshift-dependent apparent magnitude $\operatorname{mag}_{\text {lim }}(z)$ for each group.

\subsection{Contamination}

The red sequence technique is known to suffer from contamination. In our case, because the number count of objects per group that satisfy Eq. (1) is small (less than 100), we visually inspected each group member candidate and manually rejected galaxies that were unlikely to be physically connected to the group (e.g. higher redshift spiral galaxies). We also estimated contamination from background/foreground objects serendipitously falling in the same colour bin from random fields, i.e. fields large enough to ensure that they are, on average, empty. Using CFHTLS deep fields galaxy catalogues and applying Eq. (1) selection, we estimated the total background/foreground luminosity that adds up to the group catalogues. This estimation is consistent with what we have manually removed from our visual inspection. Therefore, we prefer removing the background contamination by eye since the method allows us to identify the contaminating galaxies, whereas a statistical approach would lead to subtraction of constant luminosity sheets across groups and a loss of positional information.

\subsection{Luminosity contours}

We build luminosity maps to help us characterise the largescale luminous properties of the group sample. The CFHTLS $i$-band image is divided into cells 20-pixels wide (corresponding to $\left.3.7^{\prime \prime}\right)$. Considering the centre of each cell, we compute the total rest-frame $i$-band luminosity $L$ by summing individual luminosities of the five closest group members, deduced from their isophotal magnitude $M$ :

$L=10^{\left(M_{\odot}-M+\mathrm{DM}+k+\mathrm{ev}\right) / 2.5}$

where $M_{\odot}$ is the Sun $i$-band absolute magnitude; DM is the distance modulus, $k$ the $k$-correction factor and ev the passive evolution correction, estimated from an elliptical template (Bruzual $\&$ Charlot 2003). This total luminosity is divided by the circular area enclosing the fifth neighbour: this gives a density within the considered cell that we express in units $L_{\odot} \mathrm{kpc}^{-2}$. The computed luminosity density map is then convolved with a Gaussian kernel of width 3 cells.

The choice for 5 neighbours is small compared to the number of catalogued group members (on average 46 members).

We found that the size of the cells does not influence the shape of the resulting luminosity contours. We checked that the distance to the fifth neighbour is always larger than cell $/ \sqrt{2}$, which means we do not undersample any region of the field.

\section{Weak gravitational lensing analysis}

We perform a one dimensional weak lensing (WL) analysis on each group with the goal of probing the projected mass distribution on large scales. This technique is well established and is widely used on galaxy cluster scales (e.g. Bardeau et al. 2007; Hoekstra 2007). The basic idea is the following: from the shape of the background lensed galaxy population, we can estimate the shear vector (or more precisely, the reduced shear, see e.g. Mellier 1999). Its tangential component (with respect to the centre of the deflector) is directly proportional to the mass distribution of the deflector, whereas its radial component is expected to be equal to zero in the case of perfect data. Given the relatively smaller mass of galaxy groups (compared to galaxy clusters), detecting weak lensing by groups of galaxies is challenging. Until now, weak lensing signals of such mass scales has been recovered only by stacking groups (Hoekstra et al. 2001; Parker et al. 2005; Mandelbaum et al. 2006). Moreover, the strength of the weak lensing signal is expected to decrease with the redshift of the deflector, thus we expect to detect a weak lensing signal only for the lower redshift groups. In any case, an upper limit on the velocity dispersion of the structures studied in this work is of interest. Because strongly lensed source redshifts are unknown, weak-lensing derived velocity dispersion upper limits constitute the only way to show that our sample contains only groups or poor clusters and no massive clusters. Indeed, an Einstein radius of $\sim 6^{\prime \prime}$ could be generated by a massive cluster lensing a low redshift background source.

\subsection{Weak lensing methodology}

We have been following the methodology developed by Bardeau et al. (2005, 2007), using the SHEARTOOL package 
Table 2. Summary of the strong lensing modelling.

\begin{tabular}{|c|c|c|c|c|c|c|c|c|}
\hline SL2S Group & $\mathrm{N \textrm {Nb }}$ & $\overline{P\left[{ }^{\prime \prime}\right]}$ & $\overline{\left.Y Y{ }^{\prime \prime}\right]}$ & $e$ &  & 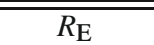 & "rms & $\overline{\chi^{2}}$ \\
\hline $\mathrm{J} 22214-0053$ & 4 & $-0.2 \pm 0.06$ & $-2.0 \pm 0.15$ & [0] & [0] & $6.83 \pm 0.15$ & 0.08 & 0.0 \\
\hline J08544-0121 & 8 & $0.0 \pm 0.1$ & $0.0 \pm 0.06$ & $0.59 \pm 0.0$ & $20.8 \pm 1.2$ & $5.48 \pm 0.12$ & 0.28 & 17 \\
\hline J09413-1100 & 4 & $-1.3 \pm 0.2$ & $-1.9 \pm 0.9$ & [0] & [0] & $7.50 \pm 0.81$ & 0.07 & 1.5 \\
\hline $\mathrm{J} 14300+5546$ & 4 & $-0.2 \pm 1.4$ & $-1.1 \pm 1.5$ & [0] & [0] & $4.69 \pm 1.43$ & 0.02 & 0.1 \\
\hline J02140-0532 & 8 & $0.85 \pm 0.3$ & $-2.6_{-0.03}^{+1.2}$ & $0.56 \pm 0.11$ & $111.2 \pm 0.1$ & $7.31 \pm 0.49$ & 0.1 & 1.1 \\
\hline J02254-0737 & 4 & $-1.9 \pm 0.15$ & $-2.6 \pm 0.6$ & {$[0]$} & {$[0]$} & $6.00 \pm 0.31$ & 0.04 & 0.4 \\
\hline J02215-0647 & 6 & $0.14 \pm 0.15$ & $-1.3 \pm 0.2$ & $0.57 \pm 0.01$ & $104 \pm 3$ & $2.56 \pm 0.14$ & 0.2 & 20 \\
\hline $\mathrm{J} 22133+0048$ & 4 & $-1.7 \pm 0.15$ & $-0.8 \pm 0.3$ & [0] & [0] & $3.40 \pm 0.2$ & 0.01 & 0 \\
\hline
\end{tabular}

Number of constraints $\mathrm{Nb}$ and optimised SIE parameters. Error bars represents $1 \sigma$ confidence level on the parameters inferred from the MCMC optimisation. Values into brackets are not optimised. This corresponds to models where the number of observational constraints is smaller than the number of free parameters characterising the SIE profile. The goodness of the fit is quantified by the RMS in the image plane and the reduced $\chi^{2}$. $e$ is the ellipticity of the mass distribution (in units $\left(a^{2}-b^{2}\right) /\left(a^{2}+b^{2}\right)$ ).

(Bardeau 2004). We refer the reader to these papers for a detailed description of the cataloguing. Here we give a brief outline of the different steps involved in the analysis of the reduced and calibrated images.

The first step is to construct a photometric catalogue for each individual image. Object positions and magnitudes were extracted with SEXTRACTOR 2.5. The second step of the analysis is to extract a star catalogue which will be used to estimate the local Point Spread Function (PSF). We select stars and clean the resulting catalogue as described in Bardeau et al. (2005). In order to measure the shapes of the stars, we used the IM2SHAPE software developed by Bridle et al. (2002). At this stage, we have a map of the PSF distribution over the entire field. The third step is to compute the galaxy catalogues selected for the weak lensing analysis. Galaxies are selected from the photometric catalogues according to the criterion described in Bardeau et al. (2005). To measure the shapes of galaxies, we first linearly interpolate the local PSF at each galaxy position by averaging the shapes of the five closest stars. The number of 5 stars is found to be large enough to locally interpolate the PSF, whereas choosing a much larger number would over-smooth the PSF characteristics. IM2SHAPE then computes intrinsic shapes of galaxies by convolving a galaxy model with the interpolated local PSF, and determines which one is the most likely by minimising residuals. In the end, IM2SHAPE's output gives a most likely model for the fitted galaxy characterised by its position, size, ellipticity and orientation, and errors on all of these quantities. For the purpose of the weak lensing analysis, we use the shape parameters derived from the CFHTLS $i$ band: observations in this photometric band have been optimised for weak lensing purposes, thus data quality in terms of seeing and source density is superior to the other bands.

Note that the reliability of the IM2SHAPE software has been validated through the simulated data of the STEP project (Heymans et al. 2006) and the results show that no significant bias is introduced by the deconvolution. It has been successfully applied in a number of weak lensing works (Kneib et al. 2003; Cypriano et al. 2004, 2005; Bardeau et al. 2005, 2007; Limousin et al. 2007a,b; Medezinski et al. 2007; Carrasco et al. 2007).

\subsection{Background Galaxies: selection and redshift distribution}

We select as background lensed sources the galaxies whose $i$-band magnitudes fall between 21.5 and 24 , hence close to the completeness magnitude for all groups (Table 3 ). The mean density of background galaxies equals to $\sim 12$ per square arcminute.
Applying the same magnitude cuts to the four CFHTLS deep fields catalogues, we find a density of $\sim 15$ per square arcminute, slightly larger than the mean density of background galaxies used in our weak lensing analysis. This is expected since we have kept only objects whose shape parameters are reliably measured, as detailed in Bardeau et al. (2005, 2007).

In order to relate the strength of the weak lensing signal to a physical velocity dispersion characterising the group potential, one needs to estimate the mean geometrical factor $D_{\mathrm{ls}} / D_{\mathrm{s}}$, where $D_{\mathrm{ls}}$ is the angular diameter distance between the lens (here the group) and the source and $D_{\mathrm{s}}$ is the angular diameter distance between the observer and the source. For this estimation, we consider the photometric redshift catalogue from the T0004 release of the CFHTLS-Deep survey, which corresponds to the deep stacks of the repeated observations of four independent MEgACAM fields. These data are therefore collected with the same instrument in the same photometric bands as the data used in this work. The CFHTLS-Deep is much deeper (at least one magnitude deeper in $r$ ) and the multicolour observations in 5 bands allow the determination of photometric redshifts. We have used the publicly available photometric redshift catalogue provided by Roser Pello ${ }^{5}$ which has been calibrated and validated with spectroscopic samples (Ienna \& Pelló 2006). We applied to this catalogue the same magnitude selection criteria that we applied to our background galaxy catalogue (i.e. $i$ between 21.5 and 24). For each group, we compute the average geometrical factor $D_{\mathrm{ls}} / D_{\mathrm{s}}$ by integrating this redshift probability distribution between 0 and 5 . We also compute the effective source redshift $z_{\text {eff }}$ that is defined by the redshift at which the geometrical factor becomes equals to the mean geometrical factor. Both quantities are given in Table 3.

Coupon et al. (2008) recently estimated photometric redshift for $35 \mathrm{deg}^{2}$ of the Wide survey. Since our group catalogue is based on a search of $100 \mathrm{deg}^{2}$, all groups do not fall in the study by Coupon et al. (2008). Moreover, they provide photometric redshifts for galaxies brighter than $i=22.5$ whereas our background galaxy catalogues contains objects as faint as $i=24$. Therefore, we cannot benefit from this work in assigning photometric redshifts individually to each background galaxy.

Coupon et al. (2008) also estimate photometric redshifts for the Deep fields down to $i=24$. A thorough comparison of both catalogues is beyond the scope of this paper, but we can check if the presented results change significantly if we use this catalogue instead of ours. We apply the same magnitude selection to the Coupon et al. (2008) catalogue and recompute the

5 http://www.ast.obs-mip.fr/users/roser/CFHTLS_TQ004/ 
quantities of interest. The main quantity we are interested in is the weak lensing inferred mass. Therefore, we present in Table 3 in brackets the masses derived using the Coupon et al. (2008) catalogue that can be compared to the masses derived using our catalogue. We find the difference between each estimate to be much smaller than the associated error bars. Therefore, we conclude that adopting one photometric catalogue or the other does not change significantly the presented results.

\subsection{Fitting the shear profile}

For each group, we look for a shear signal centred on the lens between $150 \mathrm{kpc}$ and $1.2 \mathrm{Mpc}$ from the lens. In the case of SL2S J08544-0121, a bimodal group, we use the barycentre of the light distribution instead since the lens does not dominate the light distribution. We fit an Singular Isothermal Sphere model (SIS) to the signal within this range. The choice of this model is motivated by the fact that we do not expect to get a weak lensing signal strong enough that could allow us to probe more complicated mass profiles. We use the average geometrical factor to convert the fitted Einstein radius into an SIS velocity dispersion $\sigma_{\mathrm{SIS}}$.

\section{Strong gravitational lensing analysis}

The strong lensing features can be divided in two categories. Of the 13 groups, 8 exhibit a typical "cusp" configuration where a multiply imaged system forms a characteristic arc on one side of the deflector, sometimes with a counter image on the other side of the deflector. The other 5 groups have more complex strong lensing configurations for which identification of multiple images is challenging and for which no strong lensing modelling is possible at this stage. More details on each strong lensing configuration are discussed in the Appendix.

For the 8 cusp groups, we apply a simple mass modelling in order to estimate the Einstein radius, using the LENSTOOL code (see Jullo et al. 2007, for a description of the method and how error bars are derived). The refinement of this modelling will depend on the number of available observational constraints. A realistic parametric mass modelling requires at least 5 parameters. For instance a Singular Isothermal Ellipsoid (SIE) has position $(x, y)$, ellipticity $(e)$, position angle $(\mathrm{PA})$ and Einstein radius. For a multiply imaged system where a background source is imaged $n$ times, and assuming its redshift is known, we have $2 \times(n-1)$ constraints. Only if $n \geq 4$ can we constrain an SIE profile. In the cusp sample, we have typically only one multiply imaged system, composed of 3 to 4 images which translates into 4 to 6 observational constraints. When the number of observational constraints does not allow to probe 5 free parameters, we set the ellipticity and position angle to 0 , leading to a non-elliptical mass distribution. The main robust quantity we aim to derive is the Einstein radius. For the non-cusp group, we evaluate the Einstein radius simply by measuring the distance between the strong lensing feature and the brightest group galaxy.

Optimisations are performed in the image plane, assuming a positional uncertainty equals to $0.1^{\prime \prime}$. The ellipticity of the mass distribution was forced to be smaller than 0.6 (in units $\left.\left(a^{2}-b^{2}\right) /\left(a^{2}+b^{2}\right)\right)$ as motivated by numerical simulations (Jing \& Suto 2002). Table 2 summarises information relevant to strong lensing modelling: number of constraints available; optimised parameters (position, ellipticity, position angle, Einstein radius); RMS in the image plane and reduced $\chi^{2}$, which quantifies the goodness of the fit. We note that for two systems, we are 

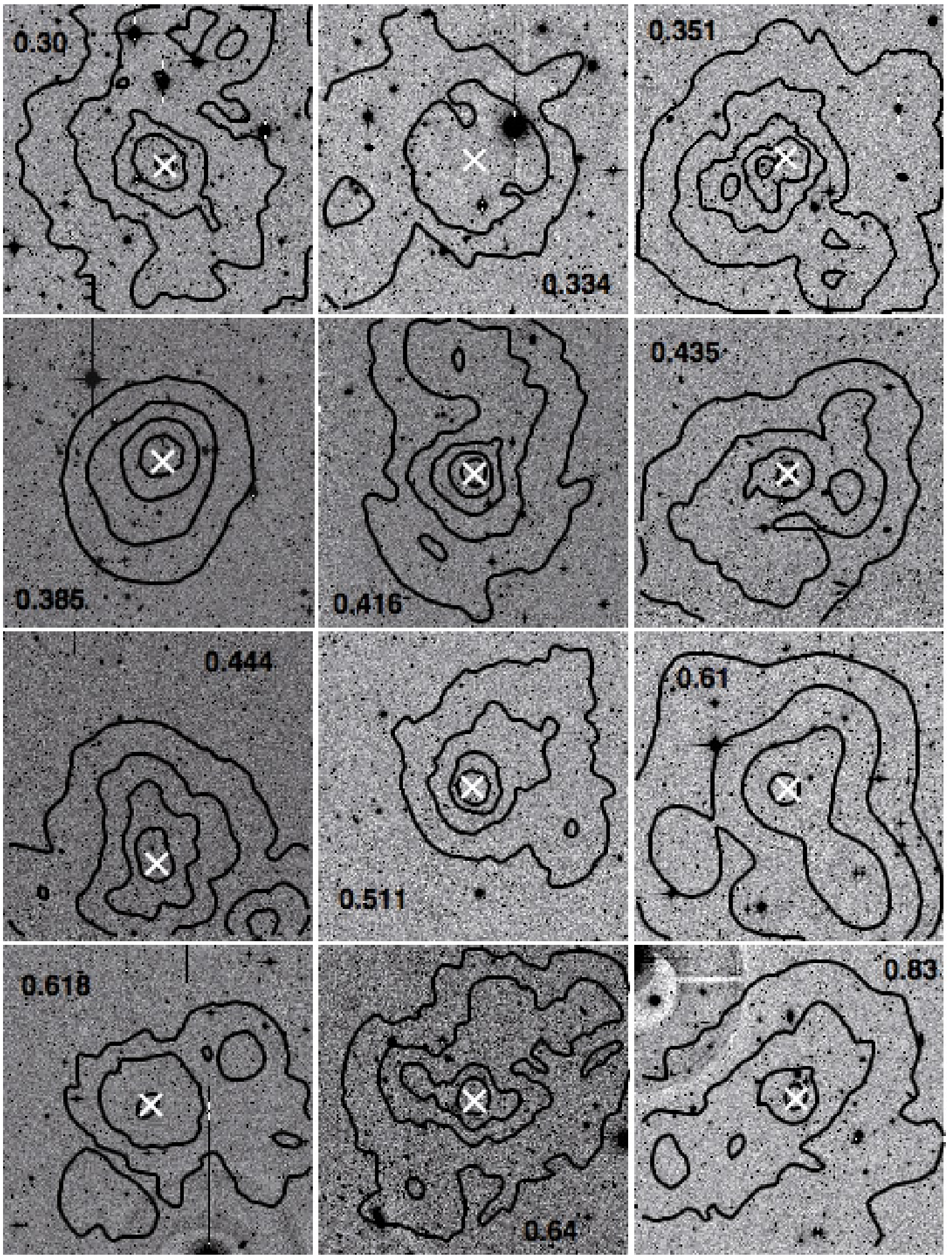

Fig. 2. Large scale $10^{\prime} \times 10^{\prime}$ CFHTLS $i$ band image for all groups but one (SL2S J08591-0345 for which data are missing). Picture are ranked by increasing redshifts as in Table 3. Redshift is reported on each picture, with three digits precision when spectroscopically measured. The white cross shows where the lens is located. Black contours correspond to luminosity contours (corrected for passive evolution) equal to $10^{5}, 3 \times 10^{5}$, $10^{6}, 3 \times 10^{6}$ and $10^{7} L_{\odot} \mathrm{kpc}^{-2}$.

unable to correctly retrieve the observational constraints, with RMS larger than the positional uncertainty: SL2S J08544-0121, $\mathrm{rms}=0.28^{\prime \prime}$ and SL2S J02215-0647, $\mathrm{rms}=0.2^{\prime \prime}$.

\section{Results}

The main properties of the groups are summarised in Table 3 and illustrated in the following figures. The name of each group 




Fig. 3. Luminosities (corrected for passive evolution) versus group redshifts.

corresponds to the equatorial coordinates (J 2000) of the brightest member. In the Appendix, we dedicate a paragraph to detail the properties of each group together with the following images:

1. a large scale $10^{\prime} \times 10^{\prime}$ CFHTLS $i$ band image. The white cross shows where the lens is located. We draw luminosity contours (corrected for passive evolution) equal to $10^{5}, 3 \times$ $10^{5}, 10^{6}, 3 \times 10^{6}$ and $10^{7} L_{\odot} \mathrm{kpc}^{-2}$;

2. a 1-square-arcminute colour image from $g, r, i$ CFHTLS imaging centred on the deflector;

3. the HST image (when available) centred on the deflector, with the image size given in the figure caption. The image is in colour when observations are available in three bands (F475, F606 and F814), and is in F606 otherwise.

Some of these visual information are summarised in Fig. 2, where we show the large scale CFHTLS images for the 12 groups (all except SL2S J08591-0345 for which data is incomplete, see Sect. 6.7).

\subsection{Einstein radii and redshift distributions}

The group sample presented here spans $z=0.30$ to $z=0.83$, with a mean redshift $\langle z\rangle=0.50$. We report in Table 3 the fitted $R_{\mathrm{E}}$ for the 8 cusp groups, as well as the measured $R_{\mathrm{E}}$ for the remaining 5 non cusp groups. They span $2.5^{\prime \prime}$ to $8^{\prime \prime}$. We expect the distribution of $R_{\mathrm{E}}$ to decrease with increasing $R_{\mathrm{E}}$ (Fig. 1). This is not seen here, but we need to correct this distribution for selection biases. Clearly we will miss more $R_{\mathrm{E}} \sim 2^{\prime \prime}$ than $R_{\mathrm{E}} \sim 5^{\prime \prime}$ systems with our detection robot (simply because a small $R_{\mathrm{E}}$ is more likely to be embedded within the light distribution of the lensing galaxy and missed for that reason).

\subsection{Luminosities}

In all cases, we find that the brightest galaxy populating the strong lensing deflector is the brightest group member. Total luminosities, corrected for passive evolution, are reported in Table 3. Figure 3 shows the luminosity as a function of redshift.

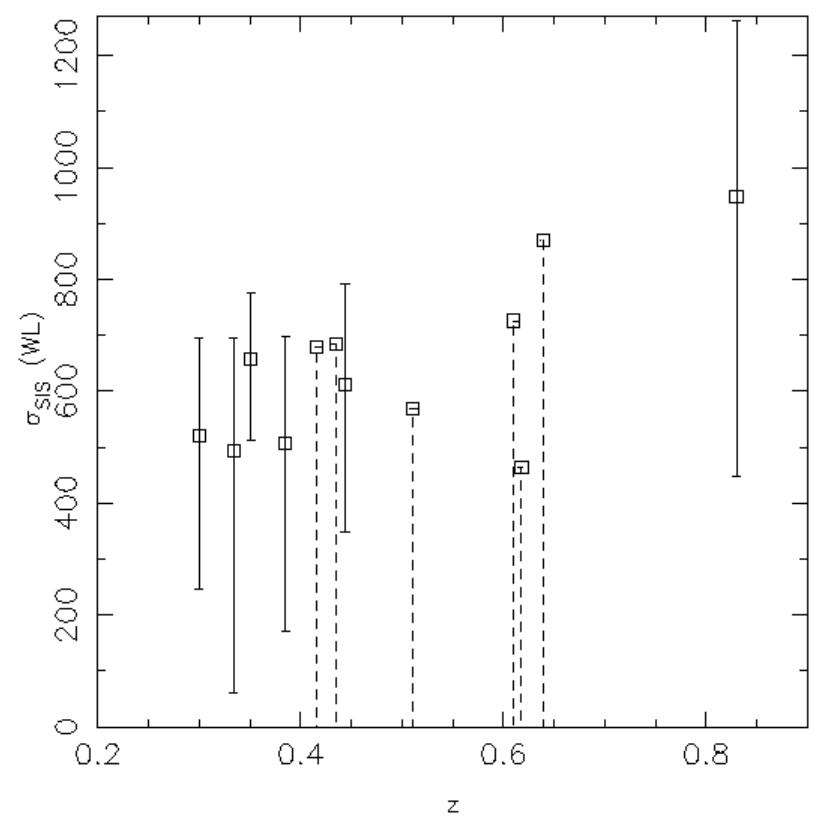

Fig. 4. Velocity dispersions inferred from fitting a Singular Isothermal Sphere to the reduced shear profile, as a function of group redshifts. Dashed lines corresponds to upper limits only.

We find that the group sample presented in this work is homogeneous in terms of total luminosities.

\subsection{Weak lensing}

We report the inferred SIS velocity dispersion for each group in Table 3 . Figure 4 shows the fitted $\sigma_{\text {SIS }}$ as a function of redshift. As expected, we see that the weak lensing constraints are tighter for the lowest redshift groups. Considering the whole sample, we see that $\sigma_{\text {SIS }} \sim 500 \mathrm{~km} \mathrm{~s}^{-1}$ with large error bars and an upper limit of $\sim 900 \mathrm{~km} \mathrm{~s}^{-1}$. Therefore, we verify that no massive cluster with $\sigma_{\text {SIS }}>1000 \mathrm{~km} \mathrm{~s}^{-1}$, which would lens a close background source, is included in our sample and that it consists of groups or poor clusters only. Note, however, that the highest redshift structure included in the present work has a velocity dispersion between 432 and $1218 \mathrm{~km} \mathrm{~s}^{-1}$. The upper limit is therefore consistent with a galaxy cluster, see Sect. 6.8 .

We are not able, given the data at hand, to pursue a more refined 2-D weak lensing study from which we could infer the shape of the mass distribution over the field and compare more precisely with the luminosity contours. Indeed, it is already challenging to infer an SIS velocity dispersion from our 1-D analysis and error bars are large. A 2 dimensional analysis would involve more free parameters and is not feasible here.

\subsection{Mass-to-light ratios}

From the above, we are able to compute mass-to-light ratios. From the weak lensing analysis, we compute the projected mass of the group within a circular aperture of $2 \mathrm{Mpc}$ centred on the lens (except SL2S J08544-0121, a bimodal group that we centre on the barycentre of the light distribution). Then we consider the total luminosity, corrected for passive evolution. Values are given in Table 3 and illustrated in Fig. 5. The mean value is equal to $\sim 250$ ( $i$ band, solar units, corrected for passive evolution), with an upper limit of 500. 


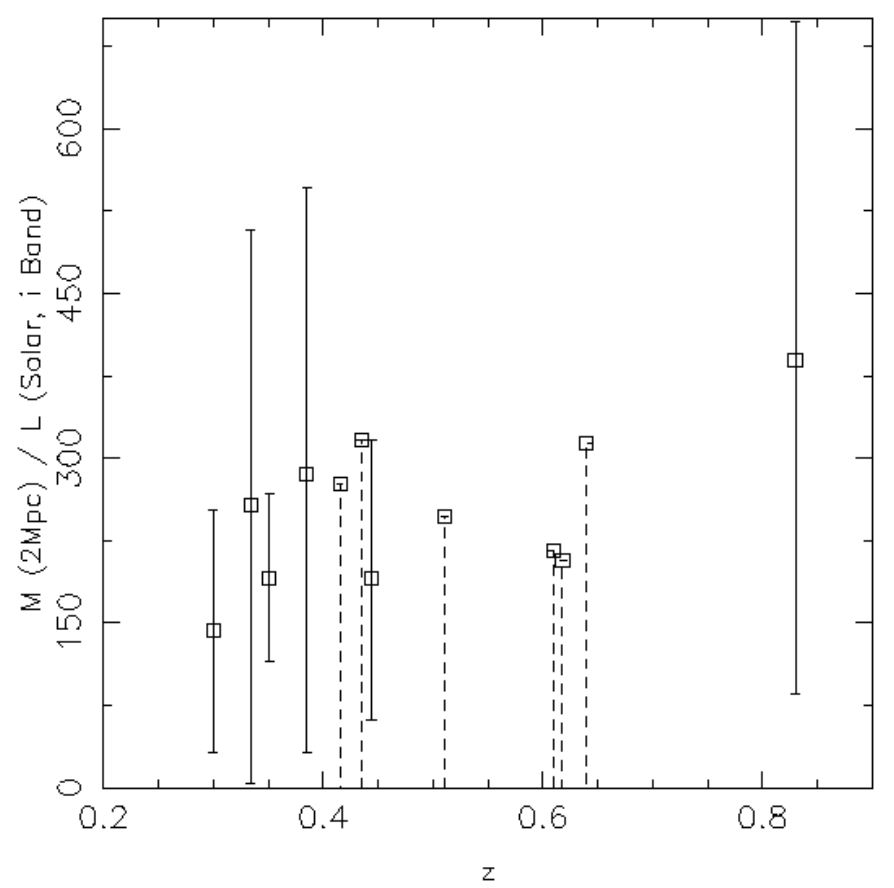

Fig. 5. Mass to Light ratio for the group sample, taking into account passive evolution correction. Dashed lines corresponds to upper limits only.

\subsection{Trends with luminosity}

If the mass is proportional to the luminosity of the systems, we expect the SIS velocity dispersion to correlate with the luminosity of the galaxy groups. We show in Fig. 6 velocity dispersions inferred from the weak lensing analysis as a function of luminosities (corrected for passive evolution). The expected trend is suggested but not statistically significant due to the large error bars.

\subsection{Number density}

We have found 13 group scale lenses within a field of $\sim 100$ square degrees (Table 1 ). This gives a rough estimate of the number density of $\sim 0.13$ groups per square arcminute. We note that some group candidates found on the CFHTLS images were not confirmed on the HST imaging and not included here. Moreover, at this stage of the project, we do not understand properly our selection function. Selection biases inherent to our detection technique exist and will be addressed in a forthcoming publication using numerical simulations. Once the SL2S will be completed and the selection function understood, we will be able to compare the distribution of observationally determined $R_{\mathrm{E}}$ to the predictions from Oguri (2006), which may provide an interesting test of the $\Lambda$ CDM scenario.

\subsection{The case of SL2S J08591-0345}

This system at $z_{\text {spec }}=0.647$ is presented in the Appendix in more detail. The observational setup makes this system special: the lens is located close $\left(75^{\prime \prime}=508 \mathrm{kpc}\right)$ to the edge of the field of view. Therefore, it is very likely that we are missing a significant part of this interesting structure (note the exotic strong lensing configuration). Therefore, we do not report a value of the luminosity for this group, neither do we pursue any weak lensing analysis.

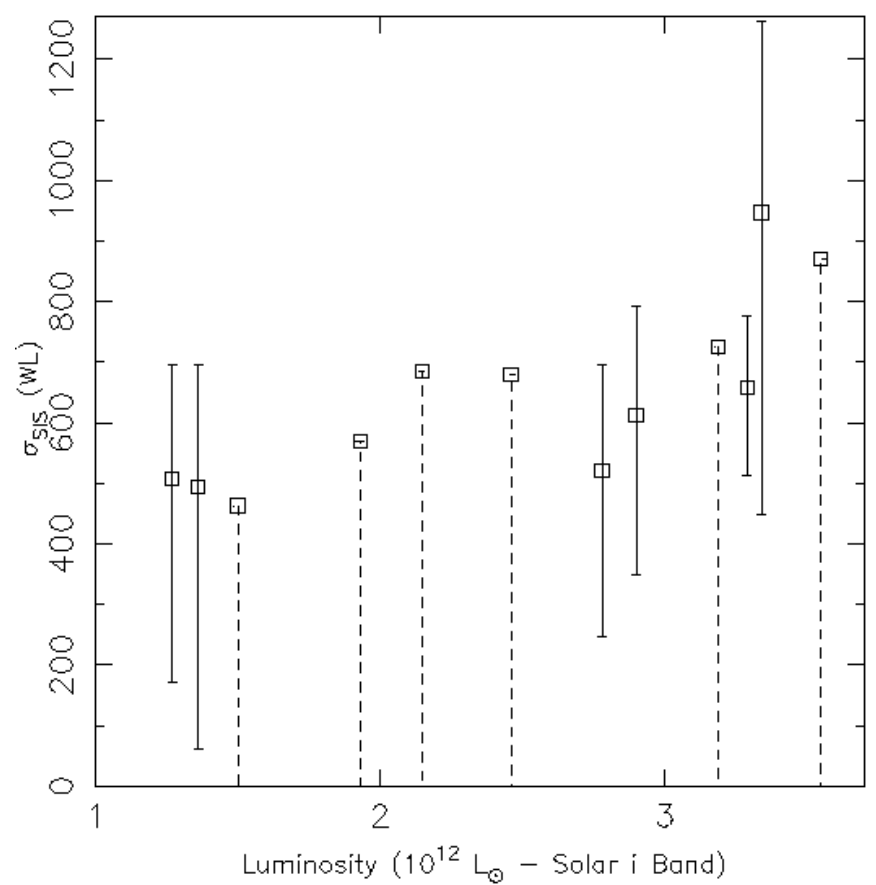

Fig. 6. SIS velocity dispersion inferred from the weak lensing analysis as a function of luminosity (corrected for passive evolution).

\subsection{The case of SL2S J22133+0048}

The weak lensing analysis of this system located at $z_{\text {phot }}=$ 0.83 yields a velocity dispersion between 432 and $1218 \mathrm{~km} \mathrm{~s}^{-1}$ which makes it consistent with a galaxy cluster. We note that its Einstein radius is rather small for a galaxy cluster, about 3.4". It is possible that SL2S J22133+0048 is indeed a galaxy cluster with a velocity dispersion larger than $1000 \mathrm{~km} \mathrm{~s}^{-1}$ lensing a close background source. An estimate of the redshift of the strongly lensed background source would help to alleviate the doubt. We also note that the redshift of this structure has been estimated photometrically. If this estimation is biased such that this structure is located at lower redshift, then we are currently overestimating its velocity dispersion.

\section{Discussion}

In this section, we discuss the results obtained on the group sample. Our statements are clearly limited by the large error bars obtained on the quantities of interest, in particular on the weak-lensing mass estimates. Therefore, part of the following discussion is qualitative only.

\subsection{Mass is traced by light}

The strong lensing system is always found to coincide with the peak of the light distribution, as can be seen in Fig. 2 (see, however, the case of SL2S J08544-0121 discussed in the Appendix). Because a strong lensing system is always associated with a significant mass concentration (occurrence of strong lensing implies $\Sigma>\Sigma_{\text {critic }}$ ), this suggests that the light is a good tracer of the mass and this justifies the choice of the strong lensing system as the group centre when we perform a weak lensing analysis. We note that this statement is limited to the fact that the centres of the luminosity and mass distributions are located approximately at the same position. We cannot say much at this point because of the limitations of the weak lensing analysis (Sect. 6.3). 


\subsection{A homogeneous sample?}

The sample is homogeneous in term of luminosities ranging between 1 and $4 \times 10^{12} L_{\odot}$ (Fig. 3). However, Fig. 2 shows that the luminosity contours have very different shapes from one group to another and that all but two (SL2S J09413-1100 at $z=0.38$ and SL2S J02254-0737 at $z=0.51$ ) have complicated shapes, suggesting that these intermediate mass structures may be dynamically young.

\subsection{Evolution with redshift?}

We looked at possible evolution of the mass-to-light ratio with redshift in Fig. 5. However, given the large error bars on this quantity, we cannot reliably infer any trend.

\subsection{Comparison to other studies}

There have been at least two different estimations of the massto-light ratio of galaxy groups from weak lensing. The first measurement of the average mass-to-light ratios was performed by Hoekstra et al. (2001) in the CNOC2 survey. They stacked the weak lensing signal obtained for 50 groups that have been selected on the basis of a dynamical analysis of group candidates. Their weak lensing detection points towards much less massive systems than the one presented in this paper: their bestfit singular isothermal sphere yields a velocity dispersion of $274_{-59}^{+48} \mathrm{~km} \mathrm{~s}^{-1}$. However, they find a mass-to-light ratio of $254 \pm$ $110 h$ (solar units, $B$ band rest frame corrected for evolution).

Subsequently, another similar study on a sample of 116 CNOC2 galaxy groups was performed by Parker et al. (2005). Given more statistics, they were able to split the group sample into subsets of poor and rich groups. Their poor galaxy groups were found to have an average velocity dispersion of $193 \pm 38 \mathrm{~km} \mathrm{~s}^{-1}$ and a mass-to-light ratio of $134 \pm 26 \mathrm{~h}$ (solar units, $B$ band rest frame), while their rich galaxy groups have a velocity dispersion of $270 \pm 39 \mathrm{~km} \mathrm{~s}^{-1}$ and a mass-to-light ratio of $278 \pm 42 h$, thus finding evidence for a steep increase in the mass-to-light ratio as a function of mass.

The groups presented in the present work are more massive than the rich galaxy group sample from Parker et al. (2005). Given the error bars obtained on the mass-to-light ratios, it is difficult to reliably compare our findings to those from Parker et al. (2005). However, considering the 6 groups for which we achieved a weak lensing detection, we get a mean velocity dispersion of $623 \mathrm{~km} \mathrm{~s}^{-1}$ and a mean mass-to-light ratio of 242 , consistent with the results of Parker et al. (2005). Note that these values are close to mass-to-light ratios of galaxy clusters (e.g. Carlberg et al. 1997; Bardeau et al. 2007).

Our results suggest that after the steep increase in mass-tolight ratio as a function of mass pointed out by Parker et al. (2005) which seems to occur at velocity dispersions around $200 \mathrm{~km} \mathrm{~s}^{-1}$ (or mass scale of order $\sim 10^{13} M_{\odot}$ ), mass-to-light ratios reach a plateau and do not evolve much from the mass scale of groups with velocity dispersions around $300 \mathrm{~km} \mathrm{~s}^{-1}$ up to rich galaxy clusters with velocity dispersion larger than $1000 \mathrm{~km} \mathrm{~s}^{-1}$. The group sample studied in this article therefore helps to bridge the gap between estimates of the mass-to-light ratio of galaxy groups with velocity dispersions in the range $200-300 \mathrm{~km} \mathrm{~s}^{-1}$ and for galaxy clusters.

\subsection{The naked cusp sample}

Eight galaxy groups reported in this article exhibit typical cusp configurations, with a clear tangential arc on one side of the deflector. For 5 of these groups, we have not been able to find the expected counter image which is usually located on the other side of the deflector, even when HST imaging was available. This incomplete cusp configuration is called "naked cusp" (see, e.g. Schneider et al. 1992; Oguri \& Keeton 2004). In two cases, SL2S J02215-0647 and SL2S J08544-0121, the counter image of the cusp is clearly identified on the HST images. In SL2S J22133+0048, the detection of the counter image is tentative only. Moreover, note that the case of SL2S J08544-0121 is a bit special since this cusp configuration is not located at the centre of the total light distribution, whereas the other cusp groups are. To summarise, we find that most of the cusp groups are naked. It may be that the current observations did not allow us to find the counter images, but this naked state of a cusp can be linked with lens statistic issue. If CDM haloes have central density slopes $<1.5$, Oguri \& Keeton (2004) predict that a significant fraction $(>20 \%)$ of large separation lenses should have naked cusp image configurations. Therefore, our results could suggest that the groups studied in this work may have shallow central density slopes.

\subsection{Other intermediate separation lenses}

We claim this work to be the first representative sample of strong lensing systems generated by group scale dark matter haloes, with intermediate image separation (in the range $\sim 3^{\prime \prime}-8^{\prime \prime}$ ). However, at least five intermediate separation lenses have been reported so far, even though the authors did not always consider the group-scale haloes as being responsible for the image separation, and are interpreting the results in terms of massive galactic scale haloes. A remarkable example is the so called Cosmic Horseshoe (Belokurov et al. 2007; Dye et al. 2008), an almost complete Einstein ring of radius $5^{\prime \prime}$ from a luminous red galaxy at $z=0.44$. The authors argued that this is the most massive galaxy lens discovered so far, with a mass enclosed within its Einstein radius of $5 \times 10^{12} M_{\odot}$, making this galaxy as massive as the entire Local Group. We rather suggest that this lensing system is simply produced by a group-scale dark-matter halo, whose centre is populated by the luminous red galaxy. Indeed, this galaxy is the brightest object in the group of $\sim 26$ members as revealed by the SDSS photometry. As suggested by the nearly perfect circle outlined by the ring, no external shear was required in the model of the system, which is not surprising because the deflector traces the centre of the group halo. This important discovery led to a more systematic data mining looking for large separation lenses in the Sloan Digital Sky Survey: the CAmbridge Sloan Survey Of Wide ARcs in the skY $\left(\right.$ CASSOWARY $\left.^{6}\right)$. Recently, Belokurov et al. (2009) reported two new large separation lenses, with Einstein radius equal to 4 " and $8^{\prime \prime}$. More recently, Lin et al. (2009), in a systematic search for bright arcs in the SDSS, reported a strongly lensed $z=2$ galaxy by a deflector whose Einstein Radius equals $3.82^{\prime \prime}$. We also mention an intermediate separation lens $\left(R_{\mathrm{E}} \sim 2.5^{\prime \prime}\right)$ found in the CLASS survey, B2108+213 (McKean et al. 2005; More et al. 2008).

We expect to find new intermediate separation lenses on completion of the CFHTLS, which, in combination with group scale lenses from other promising dedicated searches in surveys

\footnotetext{
${ }^{6}$ http://www.ast.cam.ac.uk/research/cassowary/
} 
such as the $\operatorname{SDSS}^{7}$, will increase the statistics of intermediate separation lenses, thus allowing us to get insights on this intermediate mass range.

\section{Conclusion}

We have presented a sample of 13 strong lensing features with Einstein radii between $3^{\prime \prime}$ and $8^{\prime \prime}$, i.e. generated by galaxy groupscale dark-matter haloes. This is the first representative sample (not yet complete) of intermediate image separations lenses, bridging the gap between galaxy and cluster scales. Our conclusions are the following:

1. The strong lensing analysis yields Einstein radii between $2.5^{\prime \prime}$ and $8^{\prime \prime}$.

2. We have studied the luminosity distribution to infer properties on large scales: the strong lenses coincide with the peak of the light distribution; most of the luminosity maps have complicated shapes, suggesting dynamically young structures.

3. A weak lensing analysis yields $\sigma_{\text {SIS }} \sim 500 \mathrm{~km} \mathrm{~s}^{-1}$ with large error bars and an upper limit of $\sim 900 \mathrm{~km} \mathrm{~s}^{-1}$ for the whole sample. This confirms that our sample consists of rich groups.

4. $\sigma_{\text {SIS }}$ seems to increase with luminosity but given the large error bars this is not statistically significant.

5. The mass-to-light ratio for the sample is found $M / L_{i} \sim 250$ (solar units, corrected for evolution), with an upper limit of 500 .

We note again that our conclusions are limited by the large error bars obtained on the weak lensing inferred mass estimates.

Complementary observations are ongoing: we are currently analysing multi object spectroscopy of group members as well as near infrared WIRCAM imaging. This sample will be complemented in the near future with other observations in order to help address issues of structure formation and evolution.

Once the Strong Lensing Legacy Survey will be completed and the selection function well understood, we will compare the distribution of observed Einstein radii to the predictions from Oguri (2006), which may provide an interesting test of the $\Lambda \mathrm{CDM}$ scenario.

Acknowledgements. ML acknowledges the Agence Nationale de la Recherche (Project number 06-BLAN-0067) and the Centre National d' Etudes Spatiales (CNES) for their support. The Dark Cosmology Centre is funded by the Danish National Research Foundation. JR is grateful to Caltech for its support. J.P.K. acknowledges the Centre National de la Recherche Scientifique for its support. Part of this project is done under the support of National Natural Science Foundation of China No. 10878003, 10778752, 973Program No. 2007CB815402, Shanghai Foundation No. 08240514100, 07dz22020, and the Leading Academic Discipline Project of Shanghai Normal UniversityDZL805. M.L. acknowledges Sébastien Bardeau for making its SHEARTOOL package available, and Masamune Oguri for providing the data used to generate Fig. 1. V.M. acknowledges partial support from FONDECYT through grant 1071008. We acknowledge the referee for a careful reading of the submitted paper and constructive suggestions. This research has made use of the NASA/IPAC Extragalactic Database (NED) which is operated by the Jet Propulsion Laboratory, California Institute of Technology, under contract with the National Aeronautics and Space Administration.

\section{References}

Alard, C. 2009, ArXiv e-prints 0901.0344

Auger, M. W., Fassnacht, C. D., Abrahamse, A. L., Lubin, L. M., \& Squires, G. K. 2007, AJ, 134, 668

Auger, M. W., Fassnacht, C. D., Wong, K. C., et al. 2008, ApJ, 673, 778

7 one month after this work was submitted, Kubo et al. (2008) reported the finding of five new group scale lenses in the SDSS.
Bardeau, S. 2004, Ph.D. Thesis, Université Paul Sabatier, Toulouse III, France Bardeau, S., Kneib, J.-P., Czoske, O., et al. 2005, A\&A, 434, 433 Bardeau, S., Soucail, G., Kneib, J.-P., et al. 2007, A\&A, 470, 449 Belokurov, V., Evans, N. W., Moiseev, A., et al. 2007, ApJ, 671, L9 Belokurov, V., Evans, N. W., Hewett, P. C., et al. 2009, MNRAS, 392, 104 Bertin, E., \& Arnouts, S. 1996, A\&A, 117, 393

Bolzonella, M., Miralles, J.-M., \& Pelló, R. 2000, A\&A, 363, 476

Bridle, S., Kneib, J.-P., Bardeau, S., \& Gull, S. 2002, in The shapes of galaxies and their dark halos, Proceedings of the Yale Cosmology Workshop, New Haven, Connecticut, USA, 28-30 May 2001, ed. P. Natarajan, 38 Bruzual, G., \& Charlot, S. 2003, MNRAS, 344, 1000

Cabanac, R. A., Alard, C., Dantel-Fort, M., et al. 2007, A\&A, 461, 813 Carlberg, R. G., Yee, H. K. C., \& Ellingson, E. 1997, ApJ, 478, 462 Carrasco, E. R., Cypriano, E. S., Neto, G. B. L., et al. 2007, ApJ, 664, 777 Condon, J. J., Cotton, W. D., Greisen, E. W., et al. 1998, AJ, 115, 1693 Coupon, J., Ilbert, O., Kilbinger, M., et al. 2008, ArXiv e-prints 0811.3326 Cypriano, E. S., Sodré, L. J., Kneib, J.-P., \& Campusano, L. E. 2004, ApJ, 613, 95

Cypriano, E. S., Lima Neto, G. B., Sodré, Jr., L., Kneib, J.-P., \& Campusano, L. E. 2005, ApJ, 630, 38

D’Onghia, E., Sommer-Larsen, J., Romeo, A. D., et al. 2005, ApJ, 630, L109

Dye, S., Evans, N. W., Belokurov, V., Warren, S. J., \& Hewett, P. 2008, MNRAS, 388,384

Faltenbacher, A., \& Mathews, W. G. 2007, MNRAS, 375, 313

Fassnacht, C. D., \& Lubin, L. M. 2002, AJ, 123, 627

Fassnacht, C. D., McKean, J. P., Koopmans, L. V. E., et al. 2006, ApJ, 651, 667 Faure, C., Kneib, J., Hilbert, S., et al. 2008, ArXiv e-prints 0810.4838

Feron, C., Hjorth, J., McKean, J. P., \& Samsing, J. 2008, ArXiv e-prints 0810.0780

Finoguenov, A., Ponman, T. J., Osmond, J. P. F., \& Zimer, M. 2007, MNRAS, 374,737

Gastaldello, F., Buote, D. A., Humphrey, P. J., et al. 2007, ApJ, 669, 158

Gladders, M. D., Lopez-Cruz, O., Yee, H. K. C., \& Kodama, T. 1998, ApJ, 501, 571

Grillo, C., Lombardi, M., Rosati, P., et al. 2008, A\&A, 486, 45

Helsdon, S. F., \& Ponman, T. J. 2000, MNRAS, 315, 356

Helsdon, S. F., \& Ponman, T. J. 2003, MNRAS, 339, L29

Heymans, C., Van Waerbeke, L., Bacon, D., et al. 2006, MNRAS, 368, 1323

Hoekstra, H. 2007, MNRAS, 379, 317

Hoekstra, H., Franx, M., Kuijken, K., et al. 2001, ApJ, 548, L5

Ienna, F. \& Pelló, R. 2006, in SF2A-2006: Semaine de l'Astrophysique

Francaise, ed. D. Barret, F. Casoli, G. Lagache, A. Lecavelier, \& L. Pagani, 347

Inada, N., Oguri, M., Shin, M.-S., et al. 2009, ApJ, 696, 1319

Jing, Y. P., \& Suto, Y. 2002, ApJ, 574, 538

Jullo, E., Kneib, J.-P., Limousin, M., et al. 2007, New J. Phys., 9, 447

Kassiola, A., Kovner, I., \& Blandford, R. D. 1992, ApJ, 396, 10

King, L. J. 2007, MNRAS, 956

Kneib, J., Hudelot, P., Ellis, R. S., et al. 2003, ApJ, 598, 804

Kovner, I. 1987, ApJ, 321, 686

Kubo, J. M., Allam, S. S., Annis, J., et al. 2008, ArXiv e-prints 0812.3934

Kundic, T., Hogg, D. W., Blandford, R. D., et al. 1997, AJ, 114, 2276

Limousin, M., Kneib, J. P., Bardeau, S., et al. 2007a, A\&A, 461, 881

Limousin, M., Richard, J., Jullo, E., et al. 2007b, ApJ, 668, 643

Lin, H., Buckley-Geer, E., Allam, S. S., et al. 2009, ApJ, 699, 1242

Mamon, G. A. 2007, in Groups of Galaxies in the Nearby Universe, ed. I. Saviane, V. D. Ivanov, \& J. Borissova, 203

Mandelbaum, R., Seljak, U., Cool, R. J., et al. 2006, MNRAS, 372, 758

McKean, J. P., Browne, I. W. A., Jackson, N. J., et al. 2005, MNRAS, 356, 1009

Medezinski, E., Broadhurst, T., Umetsu, K., et al. 2007, ApJ, 663, 717

Mellier, Y. 1999, ARA\&A, 37, 127

Möller, O., Natarajan, P., Kneib, J.-P., \& Blain, A. W. 2002, ApJ, 573, 562

Momcheva, I., Williams, K., Keeton, C., \& Zabludoff, A. 2006, ApJ, 641, 169

More, A., McKean, J. P., Muxlow, T. W. B., et al. 2008, MNRAS, 384, 1701

Morgan, N. D., Kochanek, C. S., Pevunova, O., \& Schechter, P. L. 2005, AJ, 129, 2531

Newton, E. R., Marshall, P. J., \& Treu, T. 2008, ArXiv e-prints 0810.3934

Oguri, M. 2006, MNRAS, 367, 1241

Oguri, M., \& Keeton, C. R. 2004, ApJ, 610, 663

Oguri, M., Keeton, C. R., \& Dalal, N. 2005, MNRAS, 364, 1451

Osmond, J. P. F., \& Ponman, T. J. 2004, MNRAS, 350, 1511

Parker, L. C., Hudson, M. J., Carlberg, R. G., \& Hoekstra, H. 2005, ApJ, 634, 806

Rasmussen, J., \& Ponman, T. J. 2007, MNRAS, 380, 1554

Schneider, P., Ehlers, J., \& Falco, E. E. 1992, Gravitational Lenses (Berlin: Springer-Verlag)

Sommer-Larsen, J. 2006, MNRAS, 369, 958

Sun, M., Voit, G. M., Donahue, M., et al. 2009, ApJ, 693, 1142 
Treu, T., Gavazzi, R., Gorecki, A., et al. 2009, ApJ, 690, 670

Trott, C. M., Treu, T., Koopmans, L. V. E., \& Webster, R. L. 2008, ArXiv e-prints 0812.0748

Tu, H., Limousin, M., Fort, B., et al. 2008, MNRAS, 1169

van den Bosch, F. C., Pasquali, A., Yang, X., et al. 2008, ArXiv e-prints 0805.002

Williams, K. A., Momcheva, I., Keeton, C. R., Zabludoff, A. I., \& Lehár, J. 2006, ApJ, 646, 85

Willis, J. P., Pacaud, F., Valtchanov, I., et al. 2005, MNRAS, 363, 675

Yang, X., Mo, H. J., \& van den Bosch, F. C. 2008, ApJ, 676, 248

${ }^{1}$ Laboratoire d'Astrophysique de Toulouse-Tarbes, Université de Toulouse, CNRS, 57 avenue d'Azereix, 65000 Tarbes, France

e-mail: marceau.limousin@ast.obs-mip.fr

2 Laboratoire d'Astrophysique de Marseille, UMR 6110, CNRS-

Université de Provence, 38 rue Frédéric Joliot-Curie, 13388

Marseille Cedex 13, France

${ }^{3}$ Dark Cosmology Centre, Niels Bohr Institute, University of Copenhagen, Juliane Maries Vej 30, 2100 Copenhagen, Denmark

4 CNRS, UMR 7095, Institut d'Astrophysique de Paris, 75014 Paris, France

5 UPMC Université Paris 06, UMR 7095, Institut d'Astrophysique de Paris, 75014 Paris, France

${ }^{6}$ Universidad de Valparaíso, Departamento de Física y Astronomia, Avenida Gran Bretaña 1111, Valparaíso, Chile
7 Durham University, Physics and Astronomy Department, South Road, Durham DH3 1LE, UK

8 Department of Astronomy, California Institute of Technology, 105-24, Pasadena, CA91125, USA

9 Department of Physics and Astronomy, University of Victoria, Victoria, BC, V8W 3P6, Canada

${ }^{10}$ Laboratoire d'Astrophysique de Toulouse-Tarbes, Université de Toulouse, CNRS, 14 avenue Edouard Belin, 31400 Toulouse, France

11 Herzberg Institute of Astrophysics, National Research Council, 5071 West Saanich Road, Victoria, BC V9E 2E7, Canada

${ }^{12}$ Laboratoire d'Astrophysique, Ecole Polytechnique Fédérale de Lausanne (EPFL), Observatoire de Sauverny, 1290 Versoix, Switzerland

13 Department of Physics, University of California, Santa Barbara, CA 93106, USA

14 Argelander-Institut für Astronomie, Universität Bonn, Auf dem Hügel 71, 53121 Bonn, Germany

15 Institute for Computational Cosmology, Department of Physics, Durham University, South Road, Durham DH1 3LE, UK

16 Physics Department \& Shanghai Key Lab for Astrophysics, Shanghai Normal University, 100 Guilin Road, Shanghai 200234, China

17 Observatoire de Paris, GEPI, CNRS-UMR 8111, 5 place Jules Janssen, 92195 Meudon Cedex, France 


\section{Appendix A: Presentation of each group}

We dedicate below a paragraph to each group to describe its main properties. The information are summarised in Table 3 and illustrated in the Appendix images.

\section{A.1. Group description}

SL2S J09013-0158 at $Z_{\text {phot }}=0.30$ (Fig. A.1): The luminosity distribution is elongated in the north-south direction. We report a straight arc between the two main bright central galaxies: this is a typical beak-to-beak configuration (Kassiola et al. 1992). This arc is found closer to the northern galaxy $\left(\sim 7^{\prime \prime}\right)$ than the southern one. No HST image is available for this group. A radio source has been reported by Condon et al. (1998) between the two bright central galaxies. Given the coordinates and associated errors of this radio source, it could be associated with one of the two smaller central galaxies.

SL2S J22214-0053 at $z_{\text {spec }}=0.334$ (Fig. A.2): The SDSS provides a redshift measurement for the main galaxy populating the deflector of 0.334 , and a velocity dispersion of $281 \pm 45 \mathrm{~km} \mathrm{~s}^{-1}$. The strong lensing deflector is populated by a single bright galaxy.

SL2S J08544-0121 at $z_{\text {spec }}=0.351$ (Fig. A.3): The strong lensing deflector is populated by a single bright galaxy whose ellipticity and position angle equals 0.3 and $25 \pm 5$ degrees, respectively. The luminosity contours are elongated in the eastwest direction and define a position angle consistent with that of the bright galaxy. We also find the position angle of the SIE halo ( $\sim 21$ degrees, Sect. 5) to be consistent with that of the bright galaxy.

Note (Fig. A.3) that the innermost luminosity contour at $10^{7} L_{\odot} \mathrm{kpc}^{-2}$ encompass the SL system but also two bright galaxies located $\sim 54^{\prime \prime}$ east from the SL system, making this light distribution bimodal. This is the only group for which the luminosity distribution is not clearly dominated by the lens, making this configuration rather exceptional: the large Einstein radius $\left(\sim 5^{\prime \prime}\right)$ points toward a massive structure associated with this lens, but the luminosity distribution is found bimodal. This suggest a dynamically young structure in the process of formation. This bright galaxy has a spectroscopic redshift measured from Keck of 0.3514 . We detect two multiply imaged systems: the brightest one is perturbed by a small satellite galaxy whose redshift is equal to 0.3517 (FORS 2); and the outer one is seen on the ACS colour image (Fig. A.3). Note how the northern counter image is found much further $\left(\sim 8^{\prime \prime}\right)$ than the main arc $\left(\sim 5^{\prime \prime}\right)$, suggesting a strong contribution from the external shear associated with the host galaxy group. The HST data brings significant additional information on this lensing feature.

SL2S J09413-1100 at $z_{\text {spec }}=0.385$ (Fig. A.4): The luminosity contours look circular. The strong lensing deflector is populated by a bright galaxy whose stellar halo is extended and presents a large ellipticity $(b / a=0.59)$ with a position angle of 74 degrees. Interestingly, this position angle is found compatible with the orientation of the luminosity contours. Note that this is the only group for which the central galaxy presents an extended stellar halo. We measured a spectroscopic redshift for this galaxy using FORS 2 to be 0.385 . We report a blue arc composed by two merging images north of the deflector, with its counter image.
We have not been able to find another counter image on the other side of the deflector, even after subtraction of the galaxy on the HST images.

SL2S J14081+5429 at $z_{\text {spec }}=0.416$ (Fig. A.5): The luminosity distribution is elongated in the north-south direction. The centre of this group is dominated by three bright galaxies aligned in the north-south direction. The brightest one $\left(\mathrm{A}, \operatorname{mag}_{i}=18.03\right)$ is the central one, that is also closest to the arc feature. North of $\mathrm{A}$ is a galaxy $\mathrm{B}\left(\mathrm{mag}_{i}=18.37\right)$ and south of is a galaxy $\mathrm{C}$ $\left(\operatorname{mag}_{i}=18.46\right)$. The SDSS provides a redshift measurement for A $(z=0.415979)$ and for B $(z=0.411022)$. A straight arc without any detected feature is located between bright central galaxies.

SL2S J14300+5546 at $z_{\text {spec }}=0.435$ (Fig. A.6): The luminosity distribution is elongated in the east-west direction. The strong lensing deflector is populated by a single galaxy whose redshift equals 0.435 (Gemini). A tangential arc is found south-east of the deflector. The position angle of the galaxy populating the deflector ( 45 degrees) is consistent with the position angle defined by the luminosity distribution.

SL2S J02140-0532 at $z_{\text {spec }}=0.444$ (Fig. A.7): The luminosity distribution is elongated in the north-south direction. The strong lensing deflector is populated by three galaxies. The two brightest ones have redshift measured spectroscopically from Keck (0.4422 and 0.4439) and may be bound gravitationally. We report an arc north of the deflector composed by two merging images showing substructure. The counter image is easily identified east of the deflector. Note that on the ground based image, we see a possible counter image south of the deflector which seems to have colour compatible with the other images. However, on the ACS data, it is clear that this feature cannot be associated with the proposed multiply imaged system. This is also confirmed by the modelling: no acceptable fit was able to reproduce the multiple images as one could have inferred from the ground based data. We note, however, that a recent independent study by Alard (2009) considers the southern image as part of the multiply imaged system. Spectroscopy of each feature is needed in order to remove the uncertainty. The HST data brings significant additional information on this lensing feature: not only does it help to identify the different images belonging to the same system, but it also resolves substructures within each lensed image, increasing

the number of constraints for the analysis. The halo of the deflector is oriented with a position angle well constrained at $111 \pm 3$ degrees. This is the same direction as the one defined by the luminosity contours.

SL2S J02254-0737 at $z_{\text {spec }}=0.511$ (Fig. A.8): The luminosity contours look circular. The strong lensing deflector is populated by a single galaxy whose redshift equals 0.511 (Gemini). We observe a tangential arc north of the lens galaxy, likely to be composed by two merging images, with an additional counter image a bit further east. The location of the deflector coincide with a radio emission reported by Condon et al. (1998).

SL2S J22130-0030 at $z_{\text {phot }}=0.61$ (Fig. A.9): The luminosity distribution is elongated in the north-south direction. The HST image reveals that the strong lensing deflector is populated by a very compact group of at least 6 galaxies. We report a blue 
arc east of the deflector, and a likely counter image presenting the same colour as the arc on the other side of the deflector.

SL2S J02215-0647 at $z_{\text {spec }}=0.618$ (Fig. A.10): We find a gap of $1.3 \mathrm{mag}$ in the $R$ band between the brightest and the second brightest galaxy, not enough to be considered as a fossil group. The strong lensing deflector is populated by a single galaxy whose redshift equals 0.618 (FORS 2). We report an arc south of the deflector, composed by two merging images. There is a counter image south-east of the deflector, as well as an additional counter image located on the other side of the deflector, resolved by HST data. We report a possible second multiply imaged system constituted by two images with same CFHTLS colours.

SL2S J14314+5533 at $z_{\text {phot }}=0.64$ (Fig. A.11): The luminosity distribution is elongated in the south-east north-west direction. A small tangential arc is found around bright galaxies. We cannot conjugate any images that may merge to form the arc. It is possible that this blue lensed feature in fact is singly imaged.

SL2S J08591-0345 at $z_{\text {spec }}=0.647$ (Fig. A.12): The lens is located close $\left(75^{\prime \prime}=508 \mathrm{kpc}\right)$ to the edge of the field of view. It is very likely that we are missing a significant part of this group. Therefore, we have not been reported any values of this group luminosity, neither did we pursue any weak lensing analysis for this group. We observe a rather exotic strong lensing configuration: the deflector is populated by three bright galaxies and two smaller ones. One of the bright one has a redshift of 0.647 (FORS 2). The multiple images draw an oval contour around the deflector. It is difficult at this point to know how many multiple images we observe and if they are coming from a single or multiple background sources. An advanced modelling of this exotic lens would be very interesting and will require deep multi colour space based data with dedicated ground based spectroscopy.

SL2S J22133+0048 at $z_{\text {phot }}=0.83$ (Fig. A.13): The luminosity distribution is elongated in the south-east north-west direction. The strong lensing deflector is populated by a single galaxy. A tangential arc composed by two merging images is found east of the deflector. A possible counter image on the other side of the deflector is detected on the space based images. The location of the deflector coincides with a radio emission reported by Condon et al. (1998). Our weak lensing analysis yields a velocity dispersion between 432 and $1218 \mathrm{~km} \mathrm{~s}^{-1}$. The upper limit is therefore consistent with a galaxy cluster. Since its Einstein radius is estimated to be about $3.4^{\prime \prime}$, it is possible that SL2S J22133+0048 is indeed a galaxy cluster with a velocity dispersion larger than $1000 \mathrm{~km} \mathrm{~s}^{-1}$ lensing a close background source. An estimate of the redshift of the strongly lensed background source would help to alleviate the doubt. We note also that the redshift of this structure has been estimated photometrically. If this estimation is biased and if this structure is located at lower redshift, then we are currently overestimating its velocity dispersion. 
M. Limousin et al.: Strong lensing by galaxy groups, Online Material $p 3$

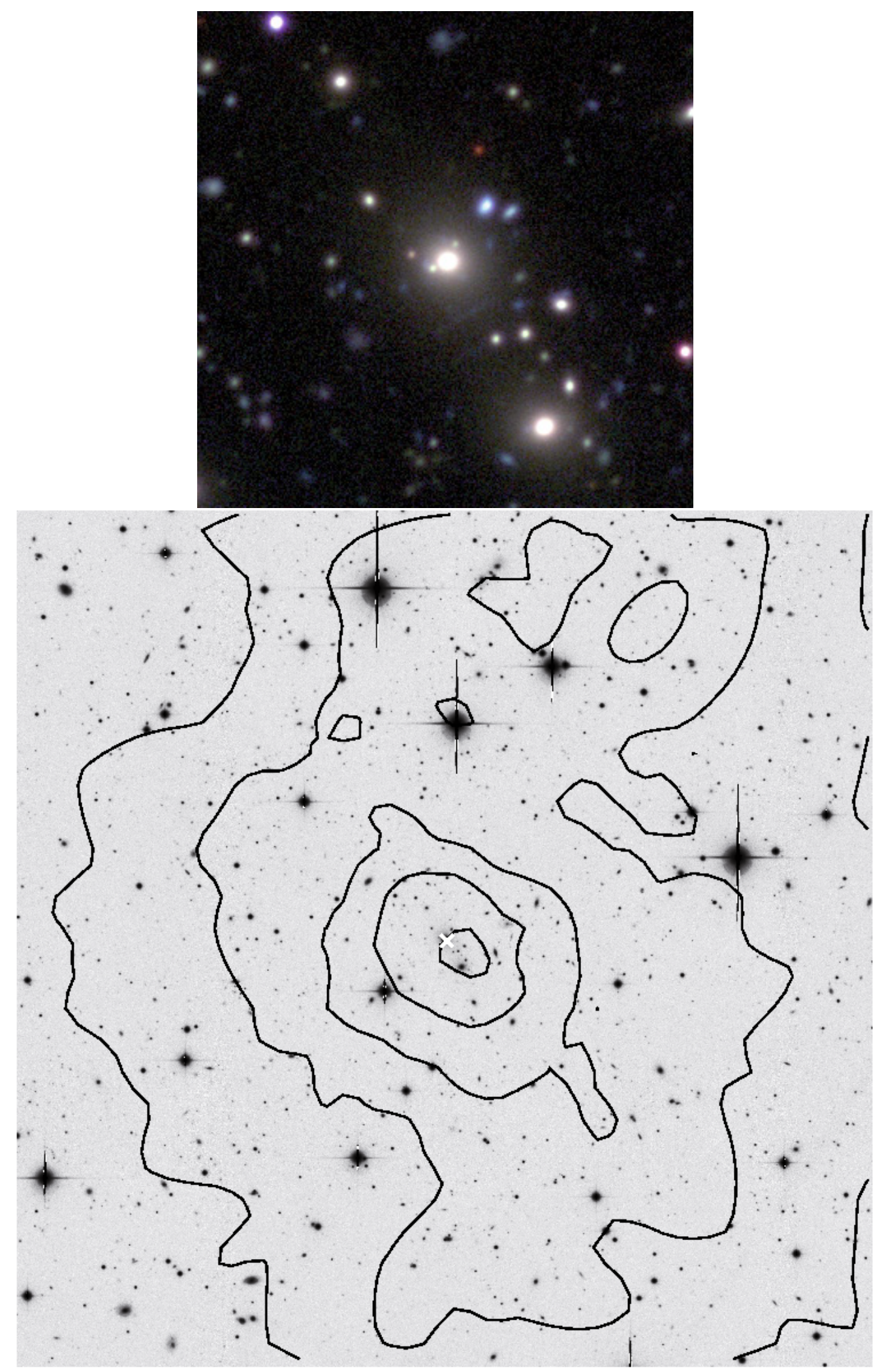

Fig. A.1. Group SL2S J09013-0158 at $z_{\text {phot }}=0.30$. Upper: composite CFHTLS $q, r, i$ colour image $\left(1\right.$ square arcminute $\left.=267 \times 267 \mathrm{kpc}^{2}\right)$. A straight arc is detected between two elliptical group members. No HST imaging is available. Lower: CFHTLS $i$ band $\left(10^{\prime} \times 10^{\prime}=2672 \times 2672 \mathrm{kpc}^{2}\right)$. We draw in black luminosity contours (corrected for passive evolution) equal to $10^{5}, 3 \times 10^{5}, 10^{6}, 3 \times 10^{6}$ and $10^{7} L_{\odot} \mathrm{kpc}^{-2}$, and the white cross shows the location of the SL system. 
M. Limousin et al.: Strong lensing by galaxy groups, Online Material p 4

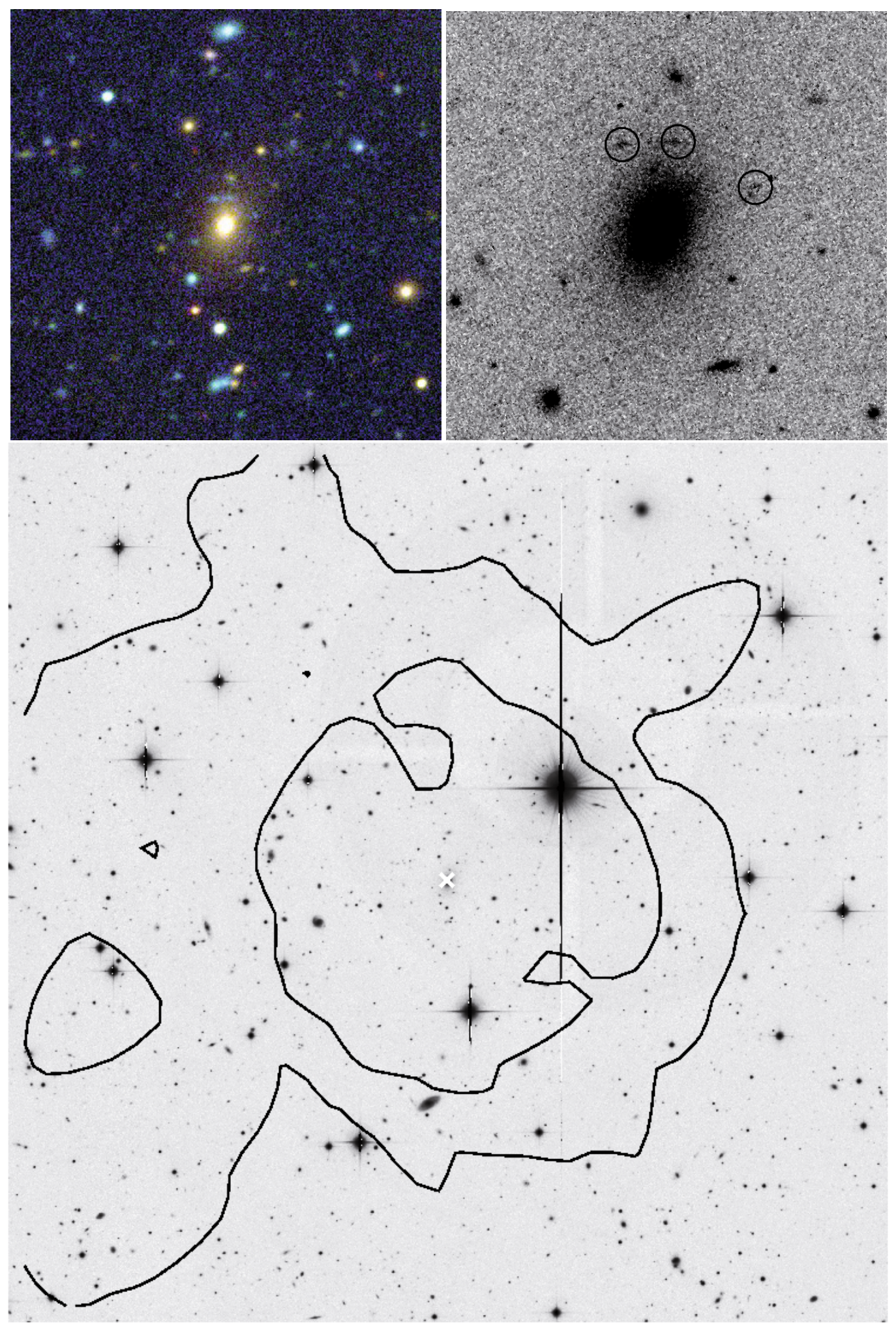

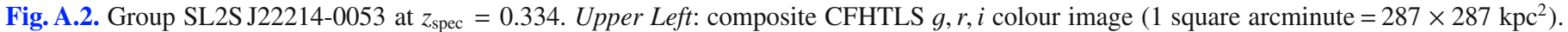
Upper Right: HST F606 image $\left(29^{\prime \prime} \times 29^{\prime \prime}=139 \times 139 \mathrm{kpc}^{2}\right)$. Lower: CFHTLS $i$ band $\left(10^{\prime} \times 10^{\prime}=2874 \times 2874 \mathrm{kpc}^{2}\right)$. White cross shows the location of the SL system, and contours are the same as in Fig. A.1. 
M. Limousin et al.: Strong lensing by galaxy groups, Online Material p 5
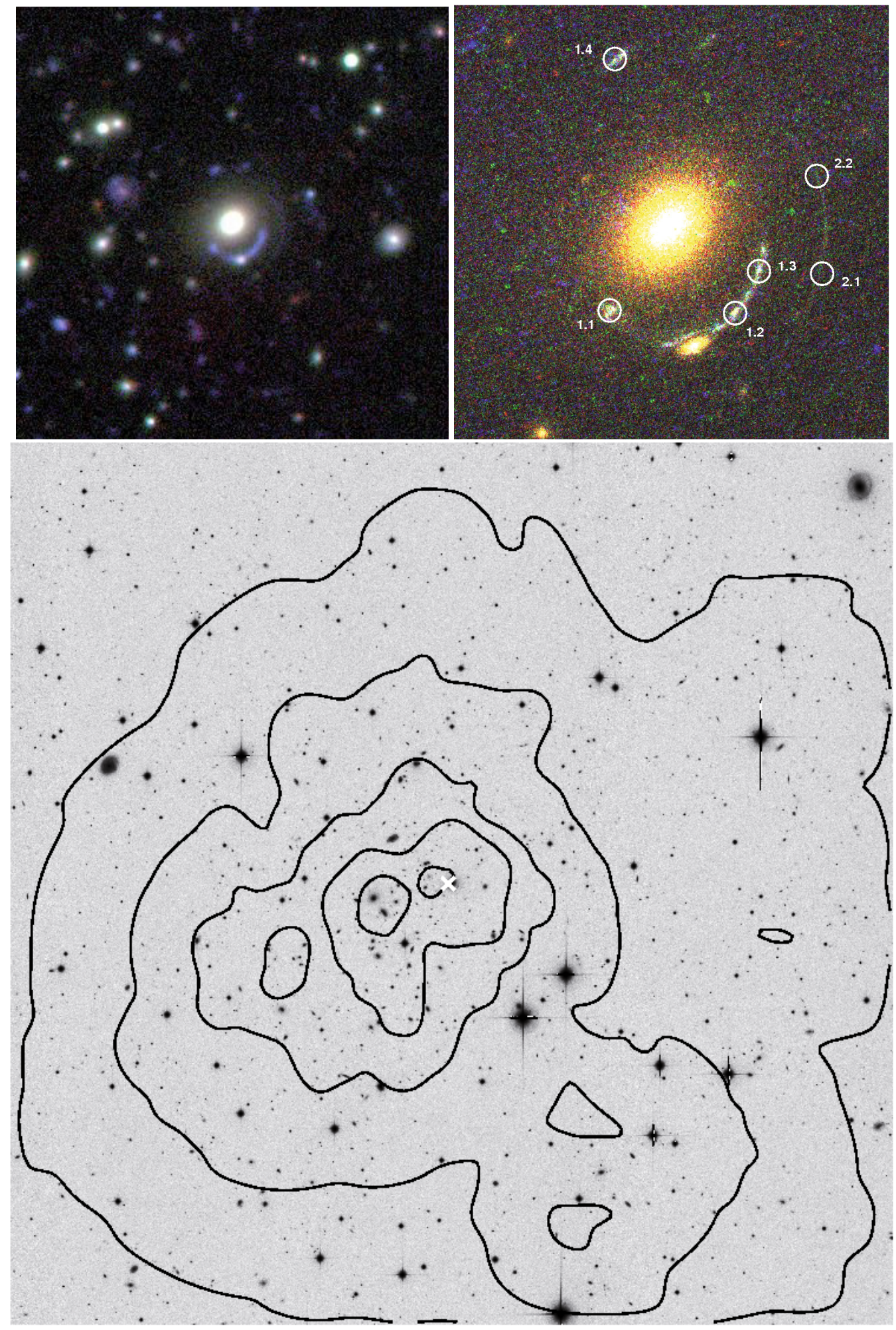


Upper Right: composite HST/ACS F814, F606, F475 colour image $\left(15^{\prime \prime} \times 15^{\prime \prime}=74 \times 74 \mathrm{kpc}^{2}\right)$. Lower: CFHTLS $i$ band $\left(10^{\prime} \times 10^{\prime}=2969 \times\right.$ $2969 \mathrm{kpc}^{2}$ ). White cross shows the location of the SL system, and contours are the same as in Fig. A.1. 
M. Limousin et al.: Strong lensing by galaxy groups, Online Material $p 6$
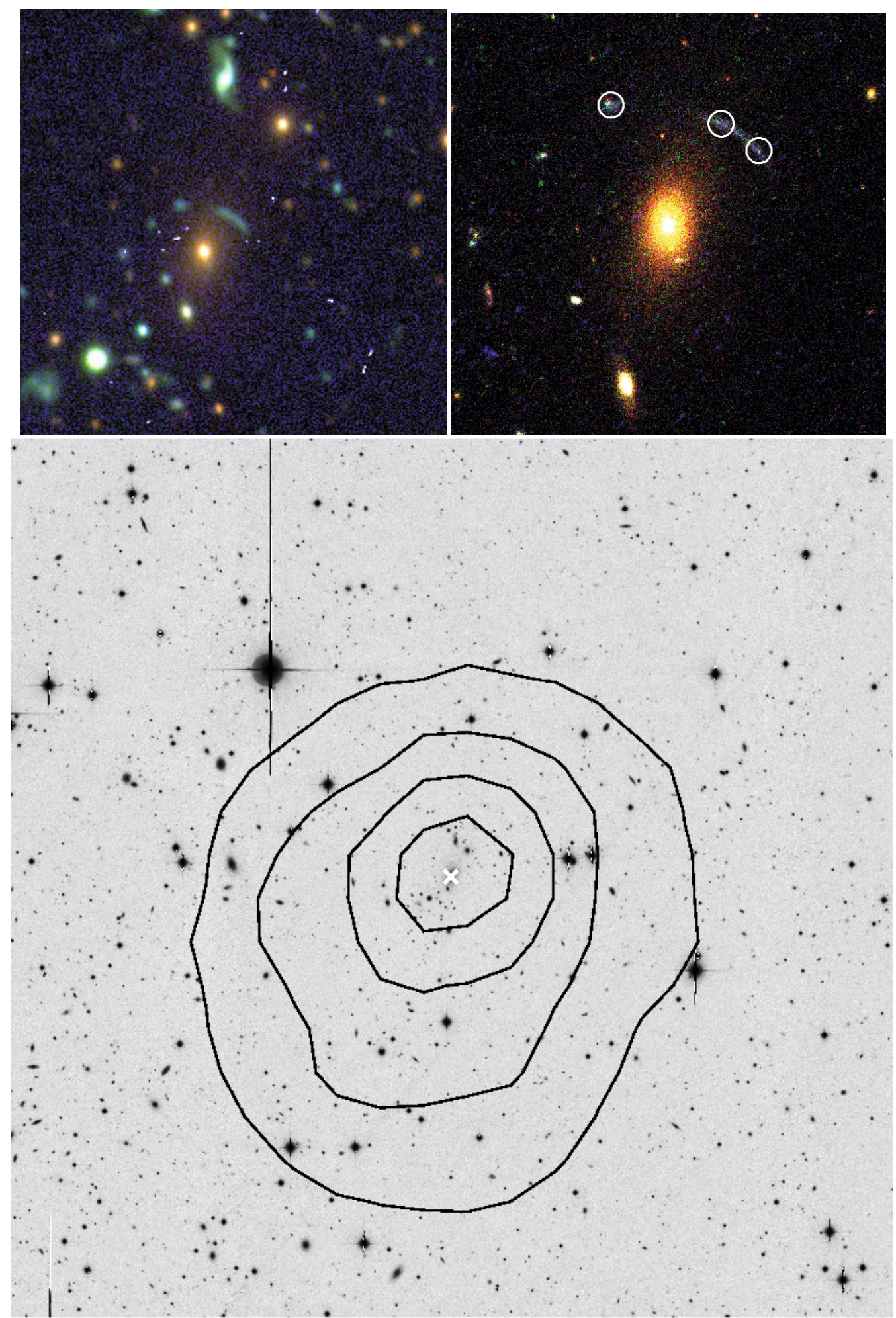

Fig. A.4. Group SL2S J09413-1100, at $z_{\mathrm{spec}}=0.385$. Upper Left: composite CFHTLS $g, r, i$ colour image $\left(1 \mathrm{square}\right.$ arcminute $\left.=315 \times 315 \mathrm{kpc}{ }^{2}\right)$. Upper Right: composite HST/ACS F814, F606, F475 colour image $\left(22^{\prime \prime} \times 22^{\prime \prime}=115 \times 115 \mathrm{kpc}^{2}\right)$. Lower: CFHTLS $i$ band $\left(10^{\prime} \times 10^{\prime}=\right.$ $3148 \times 3148 \mathrm{kpc}^{2}$ ). White cross shows the location of the SL system, and contours are the same as in Fig. A.1. 
M. Limousin et al.: Strong lensing by galaxy groups, Online Material $p$



Fig. A.5. Group SL2S J14081+5429 at $z_{\text {spec }}=0.416$. Upper Left: composite CFHTLS $g, r, i$ colour image $\left(1\right.$ square arcminute $\left.=330 \times 330 \mathrm{kpc}{ }^{2}\right)$. Upper Right: HST F606 image $\left(20^{\prime \prime} \times 20^{\prime \prime}=110 \times 110 \mathrm{kpc}^{2}\right)$. Lower: CFHTLS $i$ band $\left(10^{\prime} \times 10^{\prime}=3300 \times 3300 \mathrm{kpc}^{2}\right)$. White cross shows the location of the SL system, and contours are the same as in Fig. A.1. 
M. Limousin et al.: Strong lensing by galaxy groups, Online Material $p 8$
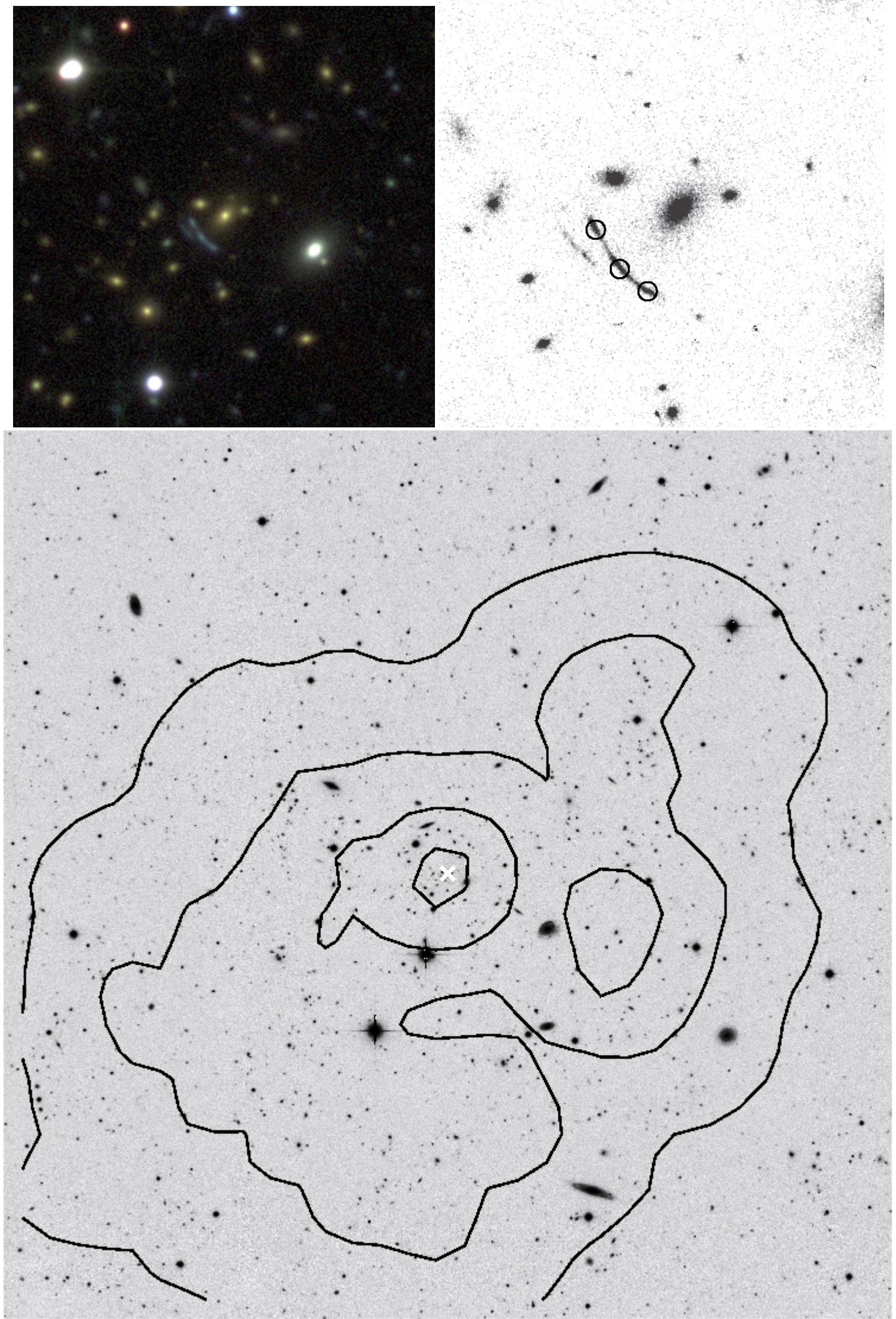

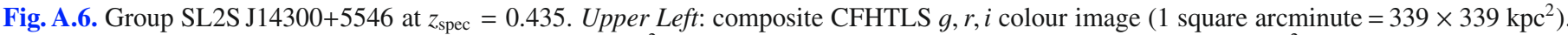
Upper Right: HST F606 image $\left(15^{\prime \prime} \times 15^{\prime \prime}=85 \times 85 \mathrm{kpc}^{2}\right)$. Lower: CFHTLS $i$ band $\left(10^{\prime} \times 10^{\prime}=3388 \times 3388 \mathrm{kpc}^{2}\right)$. White cross shows the location of the SL system, and contours are the same as in Fig. A.1. 
M. Limousin et al.: Strong lensing by galaxy groups, Online Material $p 9$

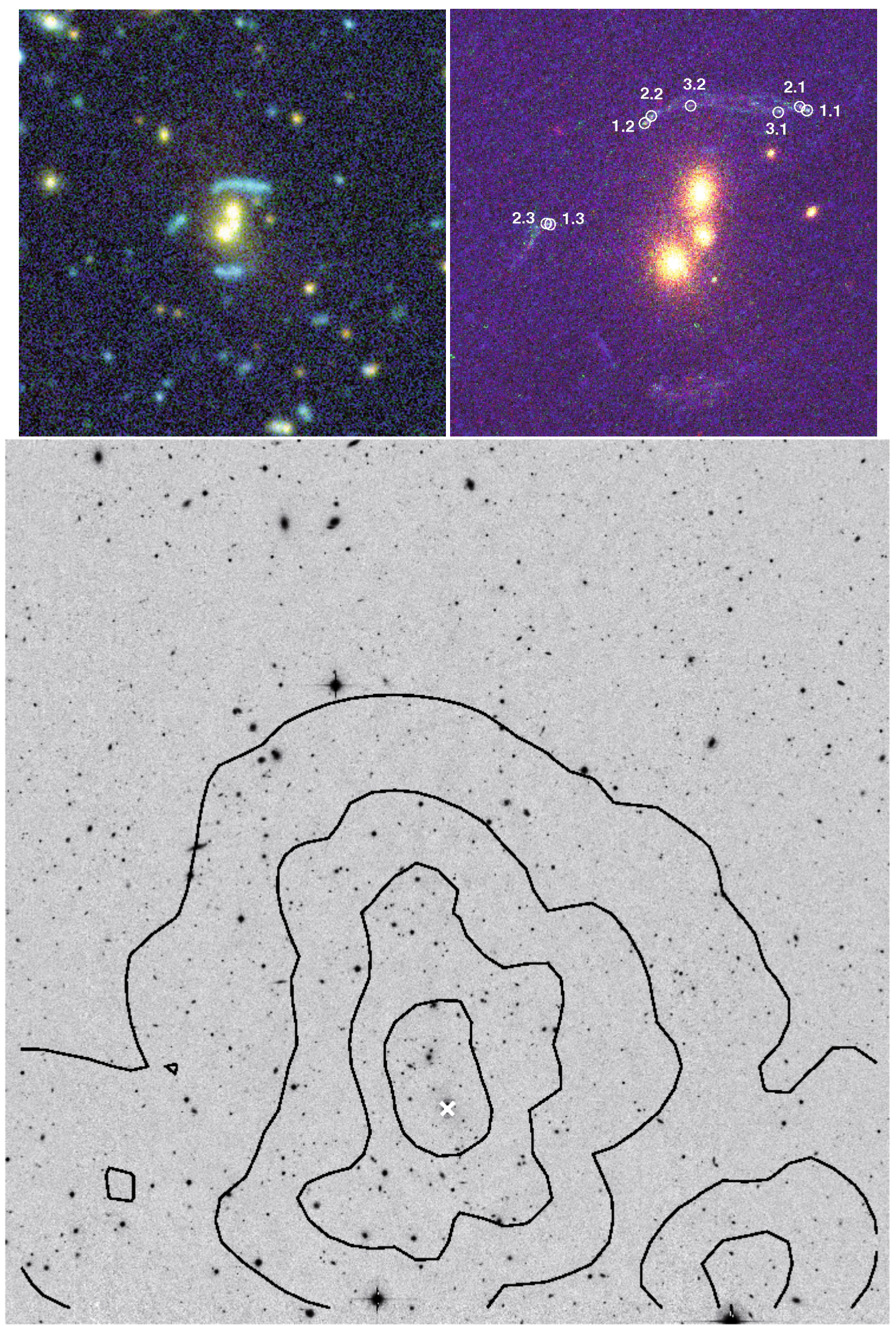

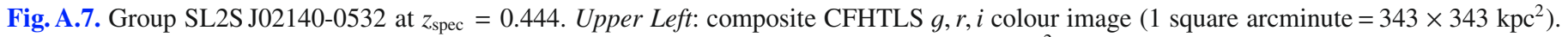
Upper Right: composite HST/ACS F814, F606, F475 colour image $\left(18^{\prime \prime} \times 18^{\prime \prime}=103 \times 103 \mathrm{kpc}^{2}\right)$. The multiply imaged system shows substructures: we conjugate three spots on the main arc and two spots on the counterimage. Note how the space based image significantly improves the identification of multiple images. Lower: CFHTLS $i$ band $\left(10^{\prime} \times 10^{\prime}=3429 \times 3429 \mathrm{kpc}^{2}\right)$. White cross shows the location of the SL system, and contours are the same as in Fig. A.1. 
M. Limousin et al.: Strong lensing by galaxy groups, Online Material p 10

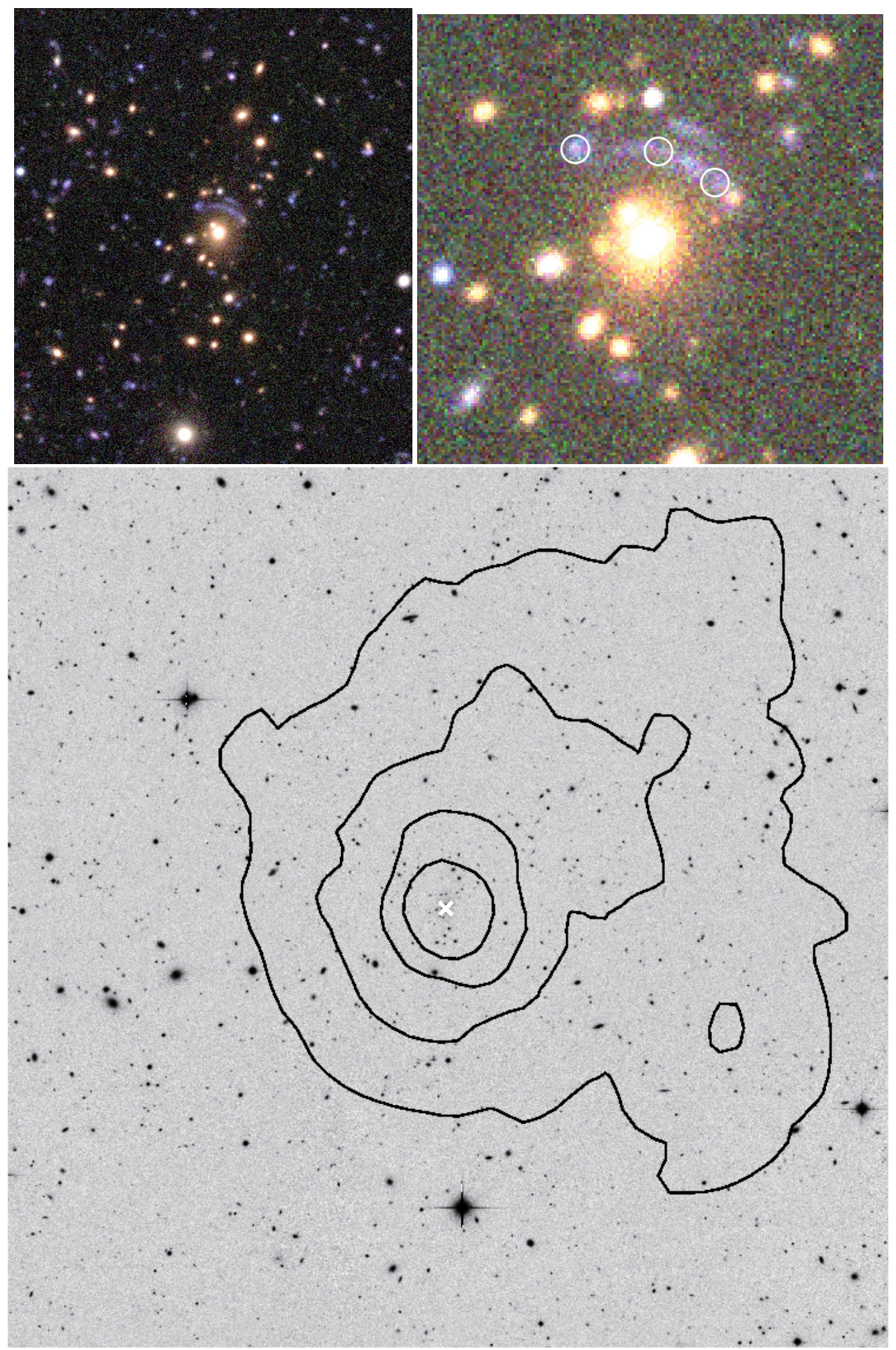


No HST imaging is available. Upper Right: composite CFHTLS $g, r, i$ colour image $\left(30^{\prime \prime} \times 30^{\prime \prime} 185 \times 185 \mathrm{kpc}^{2}\right)$ with multiple images identified. Lower: CFHTLS $i$ band $\left(10^{\prime} \times 10^{\prime}=3705 \times 3705 \mathrm{kpc}^{2}\right)$. White cross shows the location of the SL system, and contours are the same as in Fig. A.1. 
M. Limousin et al.: Strong lensing by galaxy groups, Online Material $p 11$



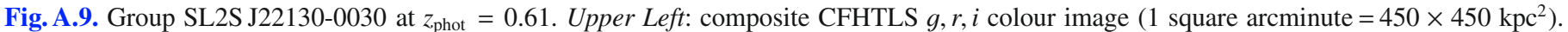
Upper Right: HST F606 image $\left(19^{\prime \prime} \times 19^{\prime \prime}=143 \times 143 \mathrm{kpc}^{2}\right)$. Lower: CFHTLS $i$ band $\left(10^{\prime} \times 10^{\prime}=4042 \times 4042 \mathrm{kpc}^{2}\right)$. White cross shows the location of the SL system, and contours are the same as in Fig. A.1. 
M. Limousin et al.: Strong lensing by galaxy groups, Online Material p 12
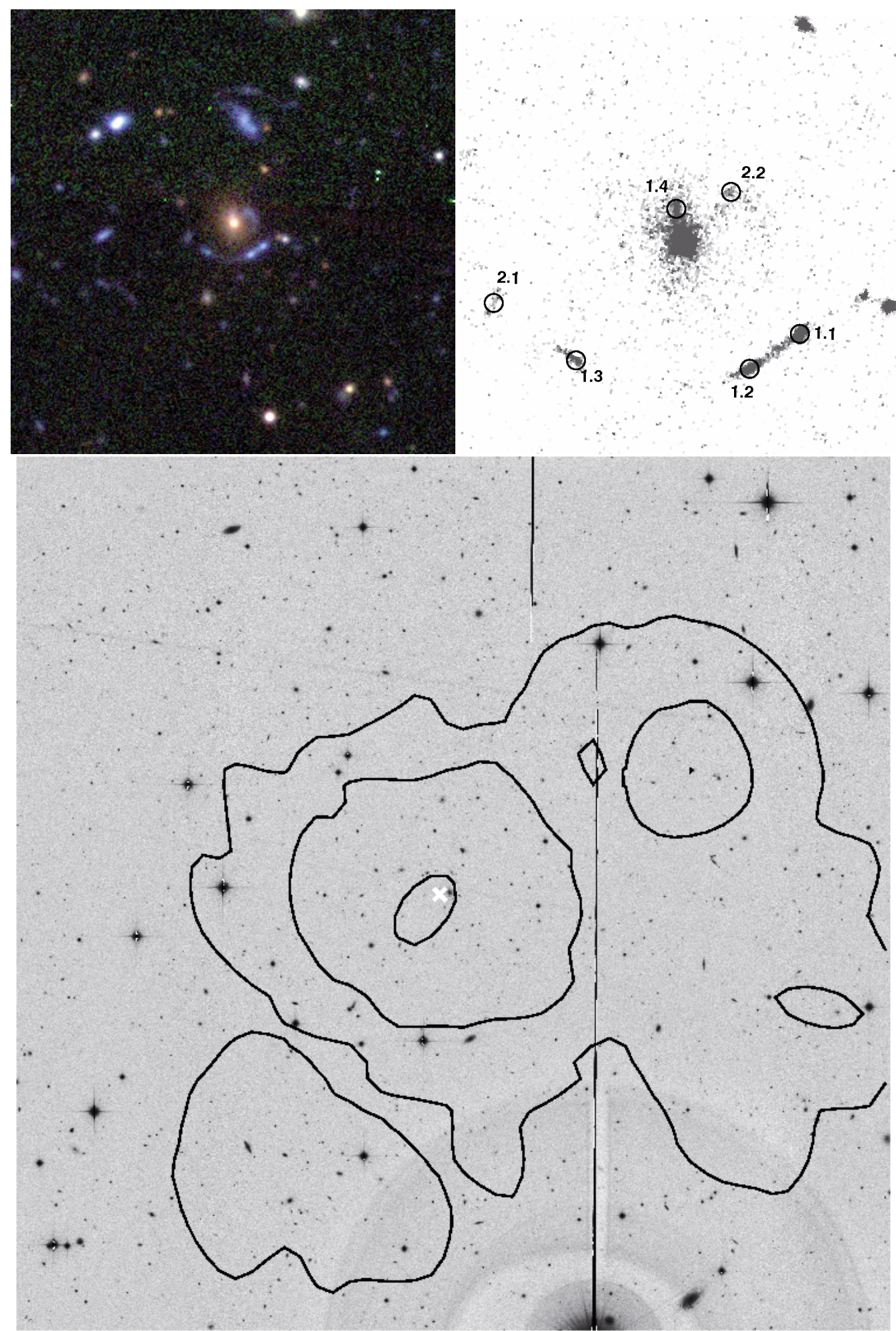

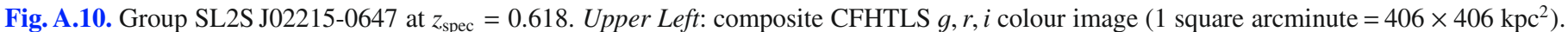
Upper Right: HST F606 image $\left(29^{\prime \prime} \times 29^{\prime \prime}=196 \times 196 \mathrm{kpc}^{2}\right)$. We propose two multiply imaged system: system 1 is a typical cusp configuration and system 2 a radial system. Lower: CFHTLS $i$ band $\left(10^{\prime} \times 10^{\prime}=4606 \times 4606 \mathrm{kpc}^{2}\right)$. White cross shows the location of the SL system, and contours are the same as in Fig. A.1. 
M. Limousin et al.: Strong lensing by galaxy groups, Online Material $p 13$

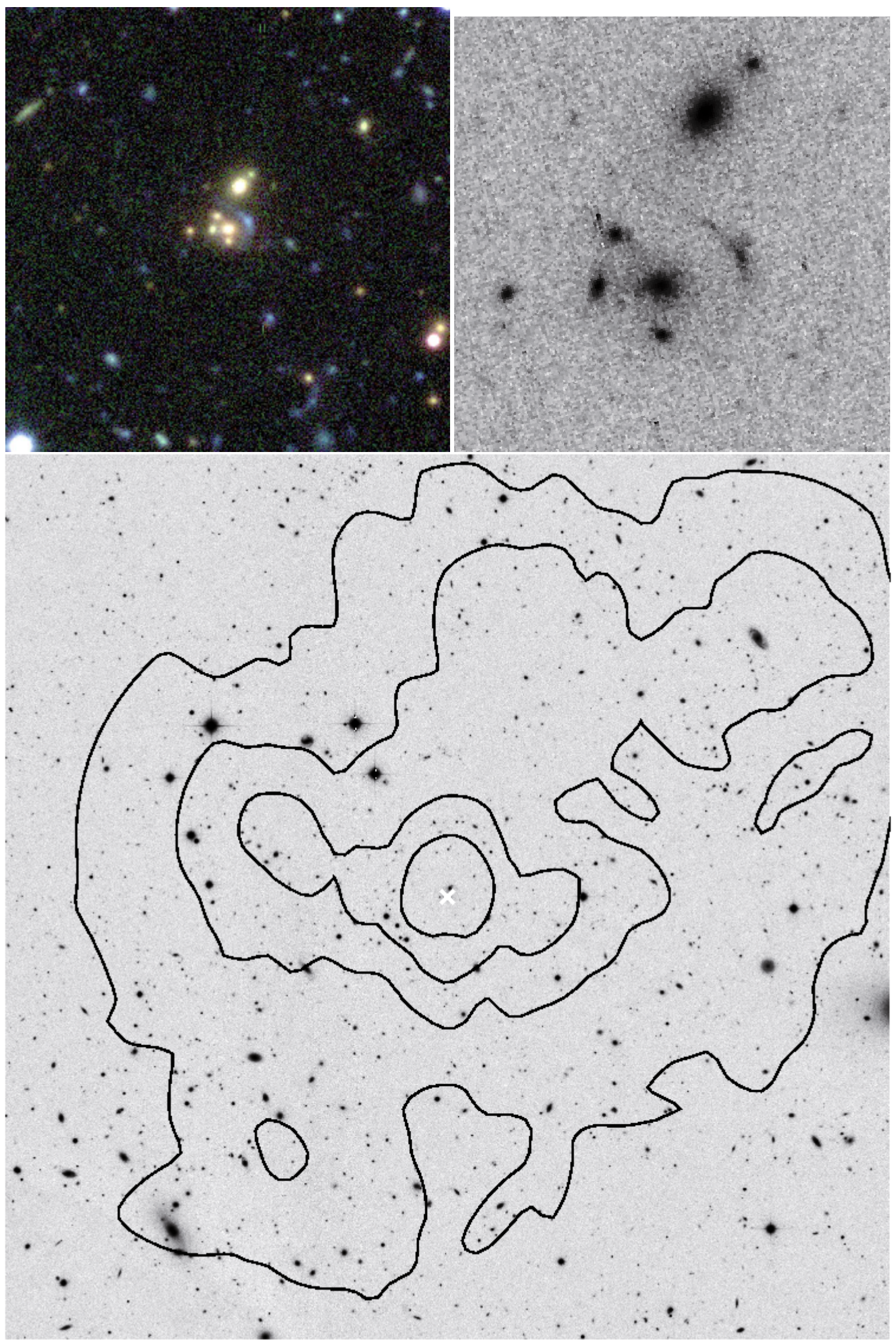

Fig. A.11. Group SL2S J14314+5533 at $z_{\text {phot }}=0.64$. Upper Left: composite CFHTLS $g, r, i$ colour image $\left(1\right.$ square arcminute $\left.=413 \times 413 \mathrm{kpc}{ }^{2}\right)$. Upper Right: HST F606 image $\left(14^{\prime \prime} \times 14^{\prime \prime}=96 \times 96 \mathrm{kpc}^{2}\right)$. Lower: CFHTLS $i$ band $\left(10^{\prime} \times 10^{\prime}=4129 \times 4129 \mathrm{kpc}^{2}\right)$. White cross shows the location of the SL system, and contours are the same as in Fig. A.1. 
M. Limousin et al.: Strong lensing by galaxy groups, Online Material p 14

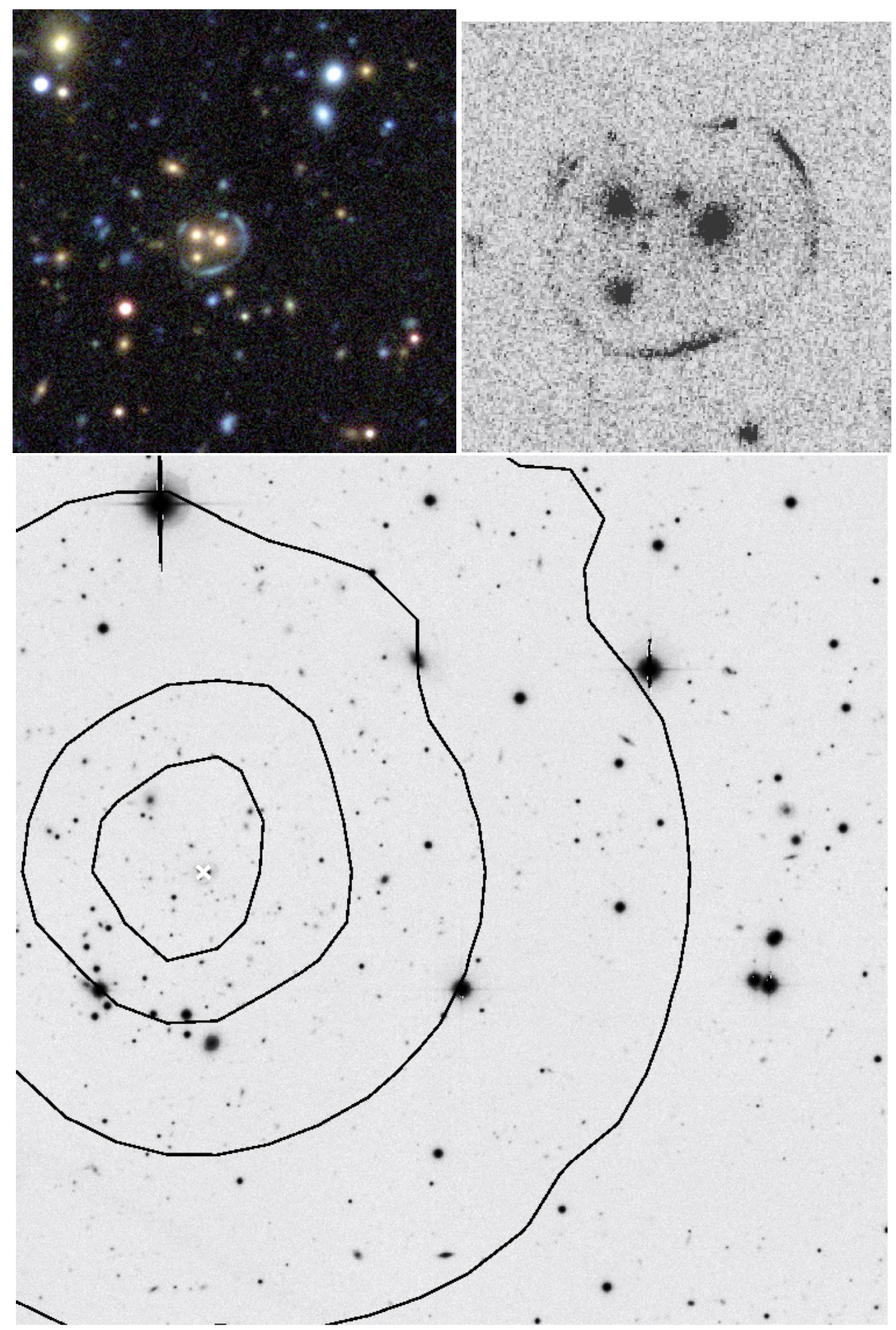

Fig. A.12. Group SL2S J08591-0345 at $z_{\text {spec }}=0.647$. Upper Left: composite CFHTLS $q, r, i$ colour image $\left(1 \mathrm{square}^{\text {arcminute }}=415 \times 415 \mathrm{kpc} \mathrm{c}^{2}\right)$. Upper Right: HST F606 image $\left(15^{\prime \prime} \times 15^{\prime \prime}=104 \times 104 \mathrm{kpc}^{2}\right)$. Lower: CFHTLS $i$ band $\left(5.3^{\prime} \times 5.3^{\prime}=2199 \times 2199 \mathrm{kpc}^{2}\right)$. Note that we miss data to probe the east part of the group. Given the observational setup, it is very likely that we miss a significant part of this galaxy group. White cross shows the location of the SL system, and contours are the same as in Fig. A.1. 
M. Limousin et al.: Strong lensing by galaxy groups, Online Material $p 15$

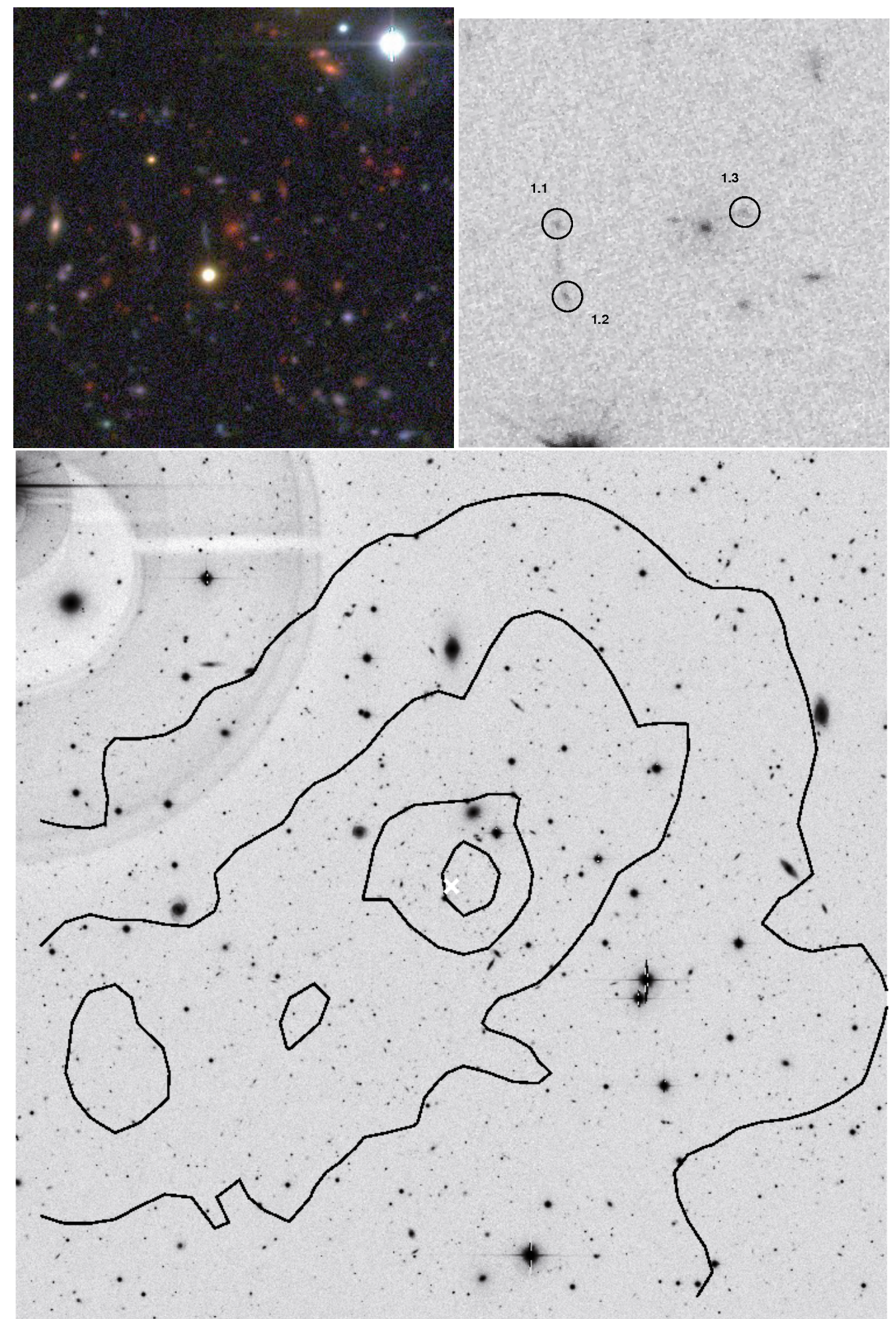

Fig. A.13. Group SL2S J22133+0048 at $z_{\text {phot }}=0.83$. Upper Left: composite CFHTLS $g, r, i$ colour image $\left(1\right.$ square $\left.\operatorname{arcminute}=456 \times 456 \mathrm{kpc}^{2}\right)$. Upper Right: HST F606 image $\left(28^{\prime \prime} \times 28^{\prime \prime}=213 \times 213 \mathrm{kpc}^{2}\right)$. Lower: CFHTLS $i$ band $\left(10^{\prime} \times 10^{\prime}=4560 \times 4560 \mathrm{kpc}^{2}\right)$. White cross shows the location of the SL system, and contours are the same as in Fig. A.1. 